\title{
SPECTRAL ANALYSIS IN A THIN DOMAIN WITH PERIODICALLY OSCILLATING CHARACTERISTICS
}

\author{
Rita Ferreira $^{1}$, M. Luísa Mascarenhas ${ }^{2}$ And Andrey Piatnitski ${ }^{3,4}$
}

\begin{abstract}
The paper deals with a Dirichlet spectral problem for an elliptic operator with $\varepsilon$-periodic coefficients in a 3D bounded domain of small thickness $\delta$. We study the asymptotic behavior of the spectrum as $\varepsilon$ and $\delta$ tend to zero. This asymptotic behavior depends crucially on whether $\varepsilon$ and $\delta$ are of the same order $(\delta \approx \varepsilon)$, or $\varepsilon$ is much less than $\delta\left(\delta=\varepsilon^{\tau}, \tau<1\right)$, or $\varepsilon$ is much greater than $\delta\left(\delta=\varepsilon^{\tau}, \tau>1\right)$. We consider all three cases.
\end{abstract}

Mathematics Subject Classification. 35P20, 49R05, 47A75, 35B27, 81Q10.

Received July 27, 2010.

Published online June 22, 2011.

\section{INTRODUCTION AND MAIN RESULTS}

When considering stationary Schrödinger's equation, the wave function $\psi$ associated to a particle in a threedimensional space is given by:

$$
-\frac{\hbar}{2 m} \Delta \psi+V \psi=E \psi
$$

where $\hbar:=h / 2 \pi, h$ being Plank's constant, $m$ is the mass of the particle, $\Delta$ is the Laplace operator, $V$ is the potential energy and $E$ is the energy of the system with wave function $\psi$.

We consider the particle confined to a certain domain $\Omega \subset \mathbb{R}^{3}$, but otherwise free; then the potential function takes the form $V(x):=0$ for $x \in \Omega$, and $V(x):=+\infty$ for $x \notin \Omega$, and the problem of finding the spatial wave function $\psi$ and the energy levels $E$ reduces to solving the following eigenvalue problem for the Laplace operator:

$$
\begin{cases}-\Delta v=\lambda v, & \text { in } \Omega, \\ v=0, & \text { on } \partial \Omega,\end{cases}
$$

where we identified $\psi \equiv v$ and $\lambda \equiv \frac{2 m}{\hbar} E$. The goal of this paper is to address the above eigenvalue problem under the assumption that the domain has a very small thickness $\delta$ and the medium presents very small $\varepsilon$-periodic

\footnotetext{
Keywords and phrases. Spectral analysis, dimension reduction, periodic homogenization, $\Gamma$-convergence, asymptotic expansions.

${ }^{1}$ I.C.T.I. - Carnegie Mellon | Portugal, F.C.T./C.M.A. da U.N.L., Quinta da Torre, 2829-516 Caparica, Portugal.

rferreir@andrew.cmu.edu; ragf@fct.unl.pt

2 Departamento de Matemática da F.C.T./C.M.A. da U.N.L., Quinta da Torre, 2829-516 Caparica, Portugal.

mascar@fct.unl.pt

${ }^{3}$ Narvik University College, P.O. Box 385, 8505 Narvik, Norway.

${ }^{4}$ P.N. Lebedev Physical Institute RAS, Leninski prospect 53, Moscow 119991, Russia. andrey@sci.lebedev.ru
} 


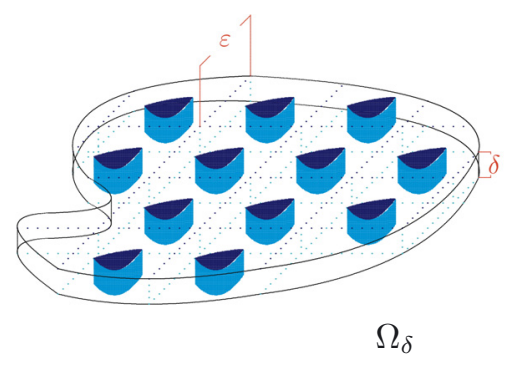

FiguRE 1. Thin and periodically oscillating media.

heterogeneities (see Fig. 1). We prove that the asymptotic behavior of the energy levels depend strongly on the ratio between small parameters $\delta$ and $\varepsilon$.

Under this motivation we consider an elliptic operator with $\varepsilon$-periodic coefficients and the corresponding Dirichlet spectral problem in a 3D bounded domain of small thickness $\delta$. We study the asymptotic behavior of spectrum of this problem as both positive parameters $\varepsilon$ and $\delta$ tend to zero. In the cases $\varepsilon \approx \delta(\delta=\varepsilon)$ and $\varepsilon \ll \delta\left(\delta=\varepsilon^{\tau}, \tau<1\right)$, the corresponding results have been announced in [8]. In the present paper we provide detailed proofs of the statements formulated in [8], and also study the case $\varepsilon \gg \delta\left(\delta=\varepsilon^{\tau}, \tau>1\right)$.

More precisely, let $\omega$ be a bounded domain in $\mathbb{R}^{2}$ and let $\delta$ be a positive parameter. Consider the thin domain $\Omega_{\delta}:=\omega \times \delta I$, where $I:=(-1 / 2,1 / 2)$. In what follows the Greek characters $\alpha$ and $\beta$ take their values in the set $\{1,2\}$ and we will often write $\bar{x}$ instead of $\left(x_{1}, x_{2}\right)$. Given a function $f: \mathbb{R}^{d} \rightarrow \mathbb{R}, d \in\{2,3\}, \bar{\nabla} f$ stands for the vector $\left(\partial f / \partial x_{1}, \partial f / \partial x_{2}\right)$, while $\nabla_{3} f$ and $\Delta_{3} f$ stand for $\partial f / \partial x_{3}$ and $\partial^{2} f / \partial x_{3}^{2}$, respectively. If $Q=\Pi_{i=1}^{d}\left(0, l_{i}\right)$ is an interval in $\mathbb{R}^{d}$, we say that $f$ is $Q$-periodic if for all $\kappa \in \mathbb{Z}$ and for a.e. $x \in \mathbb{R}^{d}$ one has $f\left(x+\kappa l_{i} e_{i}\right)=f(x)$, where $\left\{e_{i}\right\}_{i=1, \ldots, d}$ is the canonical basis of $\mathbb{R}^{d}$. A matrix is said to be $Q$-periodic if each of its components is a $Q$-periodic function.

Let $Y:=(0,1)^{2}$ and let $A=\left(a_{i j}\right)_{1 \leqslant i, j \leqslant 3} \in\left[L^{\infty}\left(\mathbb{R}^{2}\right)\right]^{3 \times 3}$ be a real, symmetric and $Y$-periodic matrix, for which there exist $\zeta, \eta \in \mathbb{R}^{+}$such that for all $\xi \in \mathbb{R}^{3}$ and for a.e. $\bar{y} \in Y$,

$$
\zeta\|\xi\|^{2} \leq(A(\bar{y}) \xi \mid \xi) \leq \eta\|\xi\|^{2} .
$$

In order to simplify the notations, we will often write $A \xi \xi$ in place of $(A \xi \mid \xi)$. For each $\varepsilon>0$ define $a_{i j}^{\varepsilon}(\bar{x}):=$ $a_{i j}\left(\frac{\bar{x}}{\varepsilon}\right)$ and $A_{\varepsilon}:=\left(a_{i j}^{\varepsilon}\right)_{1 \leqslant i, j \leqslant 3}$. Notice that, by construction, $A_{\varepsilon}$ is also a real, symmetric and $\varepsilon Y$-periodic matrix, satisfying (1.1). Our goal is to characterize the asymptotic behavior, as $\varepsilon \rightarrow 0^{+}$and $\delta \rightarrow 0^{+}$, of the eigenvalues $\lambda_{\varepsilon}^{\delta}$ associated with the spectral problem

$$
\left\{\begin{array}{l}
-\operatorname{div}\left(A_{\varepsilon} \nabla \tilde{v}_{\varepsilon}^{\delta}\right)=\lambda_{\varepsilon}^{\delta} \tilde{v}_{\varepsilon}^{\delta}, \quad \text { a.e. in } \Omega_{\delta}, \\
\tilde{v}_{\varepsilon}^{\delta} \in H_{0}^{1}\left(\Omega_{\delta}\right) .
\end{array}\right.
$$

We also assume that $a_{\alpha 3}=0$ a.e. in $\mathbb{R}^{2}$, thus we admit that the planar flux associated to the wave function depends exclusively on the behavior of this function in the cross-section $\omega$. This hypothesis enables us to decouple the limit problem, simplifying a lot our computations. We denote by $\bar{A}$ and $\bar{A}_{\varepsilon}$ the $2 \times 2$ matrices $\bar{A}:=\left(a_{\alpha \beta}\right)$ and $\bar{A}_{\varepsilon}:=\left(a_{\alpha \beta}^{\varepsilon}\right)$, respectively.

It should be noted that instead of three-dimensional thin domain one can consider problem (1.2) in a thin domain in dimension $d+1$ with $d \geq 1$. In this case $\Omega_{\delta}=\omega \times \delta I$ with $\omega \subset \mathbb{R}^{d}$. The results obtained in the paper for $d=2$ can be adapted for $d \geq 1$. We leave the details to the reader.

Since $\Omega_{\delta}$ is bounded, the spectrum $\sigma_{\varepsilon}^{\delta}$ of problem (1.2) is discrete and can be written as $\sigma_{\varepsilon}^{\delta}:=\left\{\lambda_{\varepsilon, i}^{\delta} \in \mathbb{R}^{+}\right.$: $i \in \mathbb{N}\}$, where $0<\lambda_{\varepsilon, 1}^{\delta} \leq \lambda_{\varepsilon, 2}^{\delta} \leq \cdots \leq \lambda_{\varepsilon, i}^{\delta} \leq \cdots \underset{i \rightarrow \infty}{\longrightarrow}+\infty$. As the thickness of the domain goes to zero $\left(\delta \rightarrow 0^{+}\right)$, all the eigenvalues go to infinity. A detailed characterization of the asymptotic behavior of $\sigma_{\varepsilon}^{\delta}$ is 
given in Theorem 1.1 for the case $\varepsilon \approx \delta$, in Theorem 1.2 for the case $\varepsilon \ll \delta$, and in Theorems 1.4 and 1.8 for the case $\varepsilon \gg \delta$.

Our analysis relies on $\Gamma$-convergence and asymptotic expansions techniques for spectral problems. Some of our arguments are based on the Vishik-Lusternik lemma (see [18]). For the definition and main properties of $\Gamma$-convergence we refer to [7] and to the references therein; concerning the method of asymptotic expansions in spectral problems we refer to $[4,5,18]$.

The homogenization of spectral problems, supported by a large bibliography, was first treated in [11,12,17]. The methods of analysis of spectral problems in terms of operator convergence have been elaborated in [3,16]. Other homogenization approaches in spectral problems and related topics have been studied in [1,2]. The homogenization of singularly perturbed operators have been considered in $[13,14]$ and some other works.

Consider the quadratic energy $\widetilde{E}_{\varepsilon}^{\delta}: L^{2}(\omega \times \delta I) \rightarrow[0,+\infty]$ associated with the self-adjoint operator $-\operatorname{div}\left(A_{\varepsilon} \nabla \cdot\right)$ from $L^{2}(\omega \times \delta I)$ into itself,

$$
\widetilde{E}_{\varepsilon}^{\delta}(\tilde{v}):= \begin{cases}\int_{\omega \times \delta I} A_{\varepsilon}\left(\bar{x}^{\delta}\right) \nabla \tilde{v}\left(x^{\delta}\right) \nabla \tilde{v}\left(x^{\delta}\right) \mathrm{d} x^{\delta}, & \text { if } \tilde{v} \in H_{0}^{1}(\omega \times \delta I), \\ +\infty, & \text { otherwise. }\end{cases}
$$

As it is usual in the dimension reduction framework, the first step is to perform a rescaling and a change of variables in order to transform problem (1.2) into an equivalent one defined in the fixed domain $\omega \times I$. To each point $x^{\delta}=\left(\bar{x}^{\delta}, x_{3}^{\delta}\right) \in \omega \times \delta I$ we associate the point $x=\left(\bar{x}, x_{3}\right)=\left(\bar{x}^{\delta}, \delta^{-1} x_{3}^{\delta}\right) \in \omega \times I$, and we define $v \in H_{0}^{1}(\omega \times I)$ by $v(x):=\tilde{v}\left(x^{\delta}\right)$ whenever $\tilde{v} \in H_{0}^{1}(\omega \times \delta I)$. Accordingly, we rescale the energy in (1.3) by dividing it by $\delta$ so that the new energy becomes $E_{\varepsilon}^{\delta}: L^{2}(\omega \times I) \rightarrow[0,+\infty]$,

$$
E_{\varepsilon}^{\delta}(v):= \begin{cases}\int_{\omega \times I} \bar{A}_{\varepsilon}(\bar{x}) \bar{\nabla} v(x) \bar{\nabla} v(x)+\frac{a_{33}^{\varepsilon}(\bar{x})}{\delta^{2}}\left|\nabla_{3} v(x)\right|^{2} \mathrm{~d} x, & \text { if } v \in H_{0}^{1}(\omega \times I), \\ +\infty, & \text { otherwise. }\end{cases}
$$

The rescaled spectral problem reads

$$
\left\{\begin{array}{l}
-\operatorname{div}_{\bar{x}}\left(\bar{A}_{\varepsilon} \bar{\nabla} v_{\varepsilon}^{\delta}\right)-\frac{a_{33}^{\varepsilon}}{\delta^{2}} \Delta_{3} v_{\varepsilon}^{\delta}=\lambda_{\varepsilon}^{\delta} v_{\varepsilon}^{\delta}, \text { a.e. in } \omega \times I, \\
v_{\varepsilon}^{\delta} \in H_{0}^{1}(\omega \times I)
\end{array}\right.
$$

We stress that problems (1.2) and (1.5) are equivalent.

Before stating our main results, we will introduce some notation. Since we are interested in the cases $\varepsilon \approx \delta$, $\varepsilon \ll \delta$ and $\varepsilon \gg \delta$, we consider $\delta=\varepsilon^{\tau}$ for each $\tau \in(0,+\infty)$, and we introduce the $L^{2}(Y)$-normalized first eigenpair $\left(\mu_{\varepsilon, 0}^{\tau}, \phi_{\varepsilon, 0}^{\tau}\right)$ for the bidimensional periodic spectral problem

$$
\left\{\begin{array}{l}
-\varepsilon^{2(\tau-1)} \operatorname{div}\left(\bar{A} \bar{\nabla} \phi_{\varepsilon}^{\tau}\right)+a_{33} \pi^{2} \phi_{\varepsilon}^{\tau}=\mu_{\varepsilon}^{\tau} \phi_{\varepsilon}^{\tau}, \text { a.e. in } Y, \\
\phi_{\varepsilon}^{\tau} \in H_{\#}^{1}(Y)
\end{array}\right.
$$

We recall that $C_{\#}^{\infty}(Y)$ (resp. $C_{\#}(Y)$ ) represents the subspace of $C^{\infty}\left(\mathbb{R}^{2}\right)$ (resp. $C\left(\mathbb{R}^{2}\right)$ ) of $Y$-periodic functions and $H_{\#}^{1}(Y)$ the closure of $C_{\#}^{\infty}(Y)$ with respect to the $H^{1}(Y)$-norm. Furthermore, the eigenvalue $\mu_{\varepsilon, 0}^{\tau}$ is real, positive and simple, and the associated $L^{2}(Y)$-normalized eigenfunction $\phi_{\varepsilon, 0}^{\tau}$ belongs to $H_{\#}^{1}(Y) \cap C_{\#}^{0, s}(Y)$, for some $0<s<1$, and may be chosen to be a strictly positive function (see [9]).

We will distinguish three cases: $\tau=1, \tau<1$ and $\tau>1$. Notice that if $\tau=1$ then problem (1.6) does not depend on $\varepsilon$, and for that reason we simply write $\left(\mu_{0}, \phi_{0}\right)$ to denote its $L^{2}(Y)$-normalized first eigenpair.

Let us also introduce the following unidimensional spectral problem on the interval $I$ :

$$
\left\{\begin{array}{l}
-\theta^{\prime \prime}=\varsigma \theta, \text { a.e. in } I, \\
\theta \in H_{0}^{1}(I),
\end{array}\right.
$$


whose $n$th $L^{2}(I)$-normalized eigenpair is represented by $\left(\varsigma_{n}, \theta_{n}\right)$, with $\left(\varsigma_{1}, \theta_{1}\right)=\left(\pi^{2}, \sqrt{2} \cos \left(\pi x_{3}\right)\right), x_{3} \in I$. The following statement characterizes the behavior of $\sigma_{\varepsilon}^{\delta}$ in the case $\delta \approx \varepsilon$.

Theorem $1.1(\varepsilon \approx \delta)$. Under the above hypotheses, let $\left(\lambda_{\varepsilon, k}, v_{\varepsilon, k}\right)$ be a kth eigenpair associated with problem (1.5) for $\delta=\varepsilon$, and let $\left(\nu_{k}, \varphi_{k}\right)$ be a kth eigenpair associated with the bidimensional homogenized spectral problem on the cross section $\omega$

$$
\left\{\begin{array}{l}
-\operatorname{div}\left(\bar{B}^{h} \bar{\nabla} \varphi\right)=\nu \varphi, \text { a.e. in } \omega, \\
\varphi \in H_{0}^{1}(\omega),
\end{array}\right.
$$

where the $2 \times 2$ constant matrix $\bar{B}^{h}$ is the homogenized limit of the family of $\varepsilon Y$-periodic matrices $\left\{\bar{B}_{\varepsilon}\right\}_{\varepsilon>0}$, $\bar{B}_{\varepsilon}:=\left(b_{\alpha \beta}^{\varepsilon}\right)$ with

$$
b_{\alpha \beta}^{\varepsilon}(\bar{x}):=\left[\phi_{0}\left(\frac{\bar{x}}{\varepsilon}\right)\right]^{2} a_{\alpha \beta}\left(\frac{\bar{x}}{\varepsilon}\right) .
$$

Then, there exists a self-adjoint operator $\mathcal{A}_{\varepsilon}: H_{\varepsilon} \rightarrow H_{\varepsilon}$, where $H_{\varepsilon}$ coincides algebraically with $L^{2}(\omega \times I)$ endowed with the scalar product $(\cdot \mid \cdot)_{\varepsilon}$ defined by

$$
(u \mid v)_{\varepsilon}:=\int_{\omega \times I}\left[\phi_{0}\left(\frac{\bar{x}}{\varepsilon}\right)\right]^{2} u(x) v(x) \mathrm{d} x, \quad u, v \in L^{2}(\omega \times I),
$$

such that $D\left(\mathcal{A}_{\varepsilon}\right)=H_{0}^{1}(\omega \times I)$ and

$$
\lambda_{\varepsilon, k}=\frac{\mu_{0}}{\varepsilon^{2}}+\nu_{\varepsilon, k}, \quad v_{\varepsilon, k}\left(\bar{x}, x_{3}\right)=\phi_{0}\left(\frac{\bar{x}}{\varepsilon}\right) u_{\varepsilon, k}\left(\bar{x}, x_{3}\right), \quad \text { a.e. }\left(\bar{x}, x_{3}\right) \in \omega \times I,
$$

where $\left(\nu_{\varepsilon, k}, u_{\varepsilon, k}\right)$ is a kth eigenpair of $\mathcal{A}_{\varepsilon}$, that is,

$$
u_{\varepsilon, k} \in H_{0}^{1}(\omega \times I), \quad \mathcal{A}_{\varepsilon} u_{\varepsilon, k}=\nu_{\varepsilon, k} u_{\varepsilon, k}, \quad \nu_{\varepsilon, 1} \leq \nu_{\varepsilon, 2} \leq \cdots \leq \nu_{\varepsilon, k} \leq \cdots, \quad\left(u_{\varepsilon, k} \mid u_{\varepsilon, l}\right)_{\varepsilon}=\delta_{k l} .
$$

Furthermore, $\nu_{\varepsilon, k} \rightarrow \nu_{k}$ as $\varepsilon \rightarrow 0^{+}$and, up to a subsequence that we do not relabel, $u_{\varepsilon, k} \rightarrow u_{k}$ weakly in $H_{0}^{1}(\omega \times I)$ as $\varepsilon \rightarrow 0^{+}$, where $u_{k}$ is the product of an eigenfunction associated with $\nu_{k}$ and $\theta_{1}$. Conversely, any product of eigenfunctions $u_{k}=\varphi_{k} \theta_{1}$ is the weak limit of a particular sequence of eigenfunctions associated with $\nu_{\varepsilon, k}$.

We next provide the characterization of $\sigma_{\varepsilon}^{\delta}$ when $\varepsilon \ll \delta$. For $j \in \mathbb{N}_{0}:=\mathbb{N} \cup\{0\}$, define

$$
\varrho_{j}:=\pi^{2} \int_{Y} a_{33}(\bar{y}) \psi_{j}(\bar{y}) \mathrm{d} \bar{y},
$$

where $\psi_{0} \equiv 1$ in $Y$ and, for $j \geq 1, \psi_{j}$ are the solutions of the recurrence problems in $H_{\#}^{1}(Y)$

$$
-\operatorname{div}\left(\bar{A}(\bar{y}) \bar{\nabla} \psi_{j}\right)=-a_{33}(\bar{y}) \pi^{2} \psi_{j-1}+\sum_{\ell=0}^{j-1} \varrho_{\ell} \psi_{j-1-\ell}, \quad \int_{Y} \psi_{j}(\bar{y}) \mathrm{d} \bar{y}=0 .
$$

Theorem $1.2(\varepsilon \ll \delta)$. Suppose that the above hypotheses are fulfilled and that in addition $a_{\alpha \beta}$ are uniformly Lipschitz continuous in $Y$. Let $\left(\lambda_{\varepsilon, k}, v_{\varepsilon, k}\right)$ be a kth eigenpair associated to problem (1.5) for $\delta=\varepsilon^{\tau}$ with some $\tau \in(0,1)$, and let $\left(\mu_{\varepsilon, 0}^{\tau}, \phi_{\varepsilon, 0}^{\tau}\right)$ be the $L^{2}(\omega)$-normalized first eigenpair of (1.6). Let $i \in \mathbb{N}$ be such that $\frac{i-1}{i}<\tau \leq \frac{i}{i+1}$, and let $\left(\nu_{k}, \varphi_{k}\right)$ be a kth eigenpair associated with the bidimensional homogenized spectral problem on the cross section $\omega$

$$
\left\{\begin{array}{l}
-\operatorname{div}\left(\bar{A}^{h} \bar{\nabla} \varphi\right)=\nu \varphi, \text { a.e. in } \omega \\
\varphi \in H_{0}^{1}(\omega),
\end{array}\right.
$$


where the $2 \times 2$ constant matrix $\bar{A}^{h}$ is the homogenized limit of the sequence $\left\{\bar{A}_{\varepsilon}\right\}_{\varepsilon>0}$. Then, $\mu_{\varepsilon, 0}^{\tau} \rightarrow$ $\pi^{2} \int_{Y} a_{33}(\bar{y}) \mathrm{d} \bar{y}=\varrho_{0}$ as $\varepsilon \rightarrow 0^{+}, \phi_{\varepsilon, 0}^{\tau}(\bar{x} / \varepsilon) \rightarrow 1=\psi_{0}$ uniformly in $\omega$ as $\varepsilon \rightarrow 0^{+}$, and there exists a selfadjoint operator $\mathcal{A}_{\varepsilon}: H_{\varepsilon} \rightarrow H_{\varepsilon}$, where $H_{\varepsilon}$ coincides algebraically with $L^{2}(\omega \times I)$ endowed with the scalar product $(\cdot \mid \cdot)_{\varepsilon}$ defined by

$$
(u \mid v)_{\varepsilon}:=\int_{\omega \times I}\left[\phi_{\varepsilon, 0}^{\tau}\left(\frac{\bar{x}}{\varepsilon}\right)\right]^{2} u(x) v(x) \mathrm{d} x, \quad u, v \in L^{2}(\omega \times I),
$$

such that $D\left(\mathcal{A}_{\varepsilon}\right)=H_{0}^{1}(\omega \times I)$ and

$$
\lambda_{\varepsilon, k}=\sum_{j=0}^{i} \frac{\varrho_{j}}{\varepsilon^{\tau(2 j+2)-2 j}}+\rho_{\varepsilon}^{\tau}+\nu_{\varepsilon, k}, \quad v_{\varepsilon, k}\left(\bar{x}, x_{3}\right)=\phi_{\varepsilon, 0}^{\tau}\left(\frac{\bar{x}}{\varepsilon}\right) u_{\varepsilon, k}\left(\bar{x}, x_{3}\right), \quad \text { a.e. }\left(\bar{x}, x_{3}\right) \in \omega \times I,
$$

where $\left(\nu_{\varepsilon, k}, u_{\varepsilon, k}\right)$ is a kth eigenpair of $\mathcal{A}_{\varepsilon}$, that is,

$$
u_{\varepsilon, k} \in H_{0}^{1}(\omega \times I), \quad \mathcal{A}_{\varepsilon} u_{\varepsilon, k}=\nu_{\varepsilon, k} u_{\varepsilon, k}, \quad \nu_{\varepsilon, 1} \leq \nu_{\varepsilon, 2} \leq \cdots \leq \nu_{\varepsilon, k} \leq \cdots, \quad\left(u_{\varepsilon, k} \mid u_{\varepsilon, l}\right)_{\varepsilon}=\delta_{k l} .
$$

Furthermore, $\rho_{\varepsilon}^{\tau} \rightarrow 0$ as $\varepsilon \rightarrow 0^{+}, \nu_{\varepsilon, k} \rightarrow \nu_{k}$ as $\varepsilon \rightarrow 0^{+}$, and, up to a subsequence that we will not relabel, $u_{\varepsilon, k} \rightarrow u_{k}$ weakly in $H_{0}^{1}(\omega \times I)$ as $\varepsilon \rightarrow 0^{+}$, where $u_{k}$ is the product between an eigenfunction associated with $\nu_{k}$ and $\theta_{1}$. Conversely, any product of eigenfunctions $u_{k}=\varphi_{k} \theta_{1}$ is the weak limit of a particular sequence of eigenfunctions associated with $\nu_{\varepsilon, k}$.

Remark 1.3. If the series $\sum_{j} \geqslant 0\left\|\psi_{j}\right\|_{L^{2}(Y)}$ converges, the same happens with $\sum_{j} \geqslant 0\left|\varrho_{j}\right|$ and we obtain $\sum_{j \geqslant 0} \varrho_{j}=\mu_{0}, \sum_{j \geqslant 0} \psi_{j}=\psi$, where $\psi=\phi_{0} / \int_{Y} \phi_{0} \mathrm{~d} \bar{y}$ and $\left(\mu_{0}, \phi_{0}\right)$ is the $L^{2}(Y)$-normalized first eigenpair of (1.6) for $\tau=1$. Moreover, since $\frac{i-1}{i}<\tau \leq \frac{i}{i+1}$, it can be checked that the convergence of $\sum_{j} \geqslant 0\left|\varrho_{j}\right|$ implies that, for fixed $\varepsilon>0$ and as $\tau \rightarrow 1^{-}$,

$$
\sum_{j=0}^{i} \frac{\varrho_{j}}{\varepsilon^{\tau(2 j+2)-2 j}} \rightarrow \frac{\mu_{0}}{\varepsilon^{2}}
$$

This convergence shows that, as $\tau$ approaches 1, development (1.12) approaches development (1.8) (see Appendix for the proofs).

The case $\varepsilon \gg \delta$, say $\delta=\varepsilon^{\tau}$ with $\tau \in(1,+\infty)$, seems a lot more difficult to handle due to the degeneracy of the corresponding problem (1.6). Indeed, in the case $\tau>1$ the asymptotic behavior of $\mu_{\varepsilon, 0}^{\tau}$ depends strongly on the behavior of the potential $a_{33}$ (see, for instance, $[13,14]$ ). An interesting case is when the potential $a_{33}$ oscillates between two different values, as it is the case of a two media mixture. In that direction we introduce new hypotheses on $a_{33}$. In Theorem 1.4 we identify the asymptotic expansion of the first eigenvalue. In Theorem 1.8 we provide a characterization of the limit spectrum in the sense of Kuratowsky.

Theorem 1.4 $(\varepsilon \gg \delta)$. Under the general hypotheses stated above, assume in addition that $a_{\alpha \beta}$ are smooth functions and that there exists an open and smooth subdomain $Q$ of $Y, Q \subset \subset Y$, such that $a_{33}$ coincides with its minimum, $a_{\min }$, on $Q$ and is a smooth function strictly greater than $a_{\min }$ on $Y \backslash Q$. Let $\left(\nu_{0}, q_{0}\right)$ be the $L^{2}(Q)$-normalized first eigenpair for the bidimensional spectral problem on $Q$

$$
\left\{\begin{array}{l}
-\operatorname{div}(\bar{A} \bar{\nabla} q)=\nu q, \text { a.e. in } Q, \\
q \in H_{0}^{1}(Q) .
\end{array}\right.
$$

Let $\sigma_{\varepsilon}:=\left\{\lambda_{\varepsilon, i} \in \mathbb{R}^{+}: i \in \mathbb{N}\right\}$ be the spectrum of problem (1.5) for $\delta=\varepsilon^{\tau}$ for some $\tau \in(1,+\infty)$. Let $k \in \mathbb{N}$ be such that $k \geq \frac{2}{\tau-1}$, and let $\left(\mu_{\varepsilon, 0}^{\tau}, \phi_{\varepsilon, 0}^{\tau}\right)$ be the $L^{2}(Y)$-normalized first eigenpair of (1.6). Then $\mu_{\varepsilon, 0}^{\tau} \rightarrow a_{\min } \pi^{2}$, $\phi_{\varepsilon, 0}^{\tau} \rightarrow q_{0}$ weakly in $H_{\#}^{1}(Y)$ as $\varepsilon \rightarrow 0^{+}$, where we identify $q_{0}$ with its extension by zero to the whole $Y$, and

$$
\lambda_{\varepsilon, 1}=\frac{a_{\min } \pi^{2}}{\varepsilon^{2 \tau}}+\frac{\nu_{0}}{\varepsilon^{2}}+\varepsilon^{\tau-3} \mu_{1}+\cdots+\varepsilon^{k(\tau-1)-2} \mu_{k}+\rho_{\varepsilon}^{\tau}+\nu_{\varepsilon, 1}^{\tau},
$$


where $\mu_{i}, i \in\{1, \ldots, k\}$, are well determined constants, $\left|\rho_{\varepsilon}^{\tau}\right| \leq C \varepsilon^{\left(k+\frac{1}{2}\right) \tau-\left(k+\frac{5}{2}\right)} \rightarrow 0$ as $\varepsilon \rightarrow 0^{+}$, for some constant $C$ independent of $\varepsilon$, and

$$
\nu_{\varepsilon, 1}^{\tau}:=\inf _{\substack{\psi \in H_{0}^{1}(\omega) \\\left\|\phi_{\varepsilon, 0}^{\tau}(\overline{\bar{\varepsilon}})\right\|_{L^{2}(\omega)}}}\left\{\int_{\omega}\left|\phi_{\varepsilon, 0}^{\tau}\left(\frac{\bar{x}}{\varepsilon}\right)\right|^{2} \overline{A_{\varepsilon}} \bar{\nabla} \psi \bar{\nabla} \psi \mathrm{d} \bar{x}\right\}
$$

vanishes as $\varepsilon \rightarrow 0^{+}$.

Remark 1.5. Theorem 1.4 is valid under weaker regularity hypotheses on the coefficients. In fact, as it will become clear within the proof, instead of smoothness it suffices to assume that $a_{\alpha \beta}$ are $C^{k+2}$ functions and that on $Y \backslash Q a_{33}$ is also a $C^{k+2}$ function, where $k$ is the smallest natural number satisfying $k \geq \frac{2}{\tau-1}$. In particular, the smaller $\tau-1>0$ is, the more regularity of the coefficients is required.

Remark 1.6. Hypotheses of Theorem 1.4 cover the important case where $a_{33}$ oscillates between two different values, but rule out the case where $a_{33}$ is constant. Nevertheless, it is easy to see that under the general hypotheses stated at the beginning of Section 1, if $a_{33}$ is constant, then for any $\tau \in(0,+\infty), \mu_{\varepsilon, 0}^{\tau} \equiv a_{33} \pi^{2}$ and $\phi_{\varepsilon, 0}^{\tau} \equiv 1$. Moreover, as it will become clear from our arguments, if $\left(\lambda_{\varepsilon, k}, v_{\varepsilon, k}\right)$ is a $k$ th eigenpair associated with problem (1.5) for $\delta=\varepsilon^{\tau}$, then

$$
\lambda_{\varepsilon, k}=\frac{a_{33} \pi^{2}}{\varepsilon^{2 \tau}}+\nu_{\varepsilon, k}
$$

where $\nu_{\varepsilon, k} \rightarrow \nu_{k}$ and, up to a subsequence that we do not relabel, $v_{\varepsilon, k} \rightarrow v_{k}=\varphi_{k} \theta_{1}$ weakly in $H_{0}^{1}(\omega \times I)$ as $\varepsilon \rightarrow 0^{+}$, being $\left(\nu_{k}, \varphi_{k}\right)$ a $k t h$ eigenpair associated with (1.11).

Remark 1.7. It is important to mention that the cases where $a_{33}$ is constant in $Q$, continuous in $Y$ (no jump on $\partial Q$ ) and has linear or quadratic growth in the vicinity of $\partial Q$ are very similar to the case presented here: constant in $Q$ with positive jump on $\partial Q$ and continuous on $Y \backslash Q$ (see Rem. 5.3). However, the case of an isolated minimum of $a_{33}$ is rather different and more serious modifications are required.

Finally, under quite more general hypotheses than those of Theorem 1.4, the next theorem characterizes the limit spectrum in the sense of Kuratowsky.

Theorem $1.8(\varepsilon \gg \delta)$. Assume the general hypotheses stated at the beginning of Section 1 and, in addition, assume that $a_{33}$ attains a minimum value, $a_{\min }$, at some $\bar{y}_{0} \in \mathbb{R}^{2}$ such that $a_{\alpha \beta}$ and $a_{33}$ are continuous on some neighborhood of $\bar{y}_{0}$. Then,

$$
\lim _{\varepsilon \rightarrow 0^{+}}\left(\varepsilon^{2 \tau} \sigma_{\varepsilon}\right)=\left[a_{\min } \pi^{2},+\infty\right],
$$

where the limit in (1.14) is to be understood in the sense of Kuratowsky, that is, $\left[a_{\min } \pi^{2},+\infty\right]$ is the set of all cluster points of sequences $\left\{\lambda_{\varepsilon}\right\}_{\varepsilon>0}, \lambda_{\varepsilon} \in \varepsilon^{2 \tau} \sigma_{\varepsilon}$.

The paper is organized as follows. In Section 2 we prove some auxiliary results. Section 3 is devoted to the proof of Theorem 1.1, while Section 4 to the proof of Theorem 1.2. Finally, in Section 5 we prove Theorems 1.4 and 1.8 .

\section{Preliminary Results}

In this section we present preliminary results that play an important role on the subsequent sections. The first result concerns the convergence of eigenpairs associated with a sequence of densely defined self-adjoint operators, whose proof can be found in [6], Theorem 3.1. 
Proposition 2.1. Let $\mathcal{A}_{\varepsilon}: H_{\varepsilon} \rightarrow H_{\varepsilon}$ be a sequence of densely defined self-adjoint operators, where $H_{\varepsilon}$ coincides algebraically with a fixed Hilbert space $H$ endowed with a scalar product $(\cdot \mid \cdot)_{\varepsilon}$ such that

$$
\begin{aligned}
& c_{1}\|u\|^{2} \leq(u \mid u)_{\varepsilon} \leq c_{2}\|u\|^{2}, \text { for suitable positive constants } c_{1}, c_{2}, \\
& \lim _{\varepsilon \rightarrow 0^{+}}\left(u_{\varepsilon} \mid v_{\varepsilon}\right)_{\varepsilon}=(u \mid v) \text { whenever } u_{\varepsilon} \rightarrow u \text { and } v_{\varepsilon} \rightarrow v \text { in } H \text { as } \varepsilon \rightarrow 0^{+},
\end{aligned}
$$

where $(\cdot \mid \cdot)$ stands for the scalar product in $H$ and $\|\cdot\|$ the correspondent norm. Let $G_{\varepsilon}: H \rightarrow(-\infty,+\infty]$ be defined by $G_{\varepsilon}(u):=\left(\mathcal{A}_{\varepsilon} u \mid u\right)_{\varepsilon}$, if $u \in D\left(\mathcal{A}_{\varepsilon}\right)$, and $G_{\varepsilon}(u):=+\infty$, otherwise. Assume further that the three following conditions hold:

(i) $G_{\varepsilon}(u) \geq-c_{0}\|u\|^{2}$, for a suitable constant $c_{0} \geq 0$ independent of $\varepsilon$;

(ii) If $\sup G_{\varepsilon}\left(u_{\varepsilon}\right)<+\infty$ and $\sup \left\|u_{\varepsilon}\right\|<+\infty$, then the sequence $\left\{u_{\varepsilon}\right\}_{\varepsilon>0}$ is strongly relatively compact in $H$;

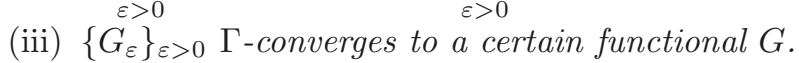

Then, the limit functional $G$ determines a unique closed linear operator $\mathcal{A}_{0}: H \rightarrow H$ with compact resolvent such that $G(u)=\left(\mathcal{A}_{0} u \mid u\right)$, for all $u \in D\left(\mathcal{A}_{0}\right)$. Furthermore, the spectral problems associated with $\mathcal{A}_{\varepsilon}$ converge in the following sense: let $\left\{\left(\nu_{\varepsilon, k}, u_{\varepsilon, k}\right)\right\}_{k \in \mathbb{N}}$ and $\left\{\left(\nu_{k}, u_{k}\right)\right\}_{k \in \mathbb{N}}$ be such that

$$
\begin{array}{clll}
u_{\varepsilon, k} \in D\left(\mathcal{A}_{\varepsilon}\right), & \mathcal{A}_{\varepsilon} u_{\varepsilon, k}=\nu_{\varepsilon, k} u_{\varepsilon, k}, & \nu_{\varepsilon, 1} \leq \nu_{\varepsilon, 2} \leq \cdots \leq \nu_{\varepsilon, k} \leq \cdots, & \left(u_{\varepsilon, k} \mid u_{\varepsilon, l}\right)_{\varepsilon}=\delta_{k l} \\
u_{k} \in D\left(\mathcal{A}_{0}\right), & \mathcal{A}_{0} u_{k}=\nu_{k} u_{k}, & \nu_{1} \leq \nu_{2} \leq \cdots \leq \nu_{k} \leq \cdots, & \left(u_{k} \mid u_{l}\right)=\delta_{k l}
\end{array}
$$

where $\delta_{k l}$ denotes the Kronecker symbol. Then $\nu_{\varepsilon, k} \rightarrow \nu_{k}$ as $\varepsilon \rightarrow 0^{+}$. Moreover, up to a subsequence that we will not relabel, $\left\{u_{\varepsilon, k}\right\}_{\varepsilon>0}$ converges as $\varepsilon \rightarrow 0^{+}$to an eigenfunction associated to $\nu_{k}$. Conversely, any eigenfunction $u_{k}$ is the strong limit of a particular sequence of eigenfunctions of $\mathcal{A}_{\varepsilon}$ associated with $\nu_{\varepsilon, k}$.

Remark 2.2. We recall that condition (iii) in Proposition 2.1 is equivalent to saying that the following two conditions are satisfied:

a) If $u_{\varepsilon}, u \in H$ are such that $u_{\varepsilon} \rightarrow u$ in $H$ as $\varepsilon \rightarrow 0^{+}$, then $G(u) \leq \liminf _{\varepsilon \rightarrow 0^{+}} G_{\varepsilon}\left(u_{\varepsilon}\right)$;

b) Given $u \in H$, there exists $\left\{u_{\varepsilon}\right\}_{\varepsilon>0} \subset H$ such that $u_{\varepsilon} \rightarrow u$ in $H$ as $\varepsilon \rightarrow 0^{+}$, and $G(u)=\lim _{\varepsilon \rightarrow 0^{+}} G_{\varepsilon}\left(u_{\varepsilon}\right)$.

The next proposition regards a classical change of unknowns (cf. [17]; see also [2]). In the cases $\varepsilon \approx \delta$ and $\varepsilon \ll \delta$ it will allow us to transform the energies (1.4) into functionals for which Proposition 2.1 applies.

Proposition 2.3. For fixed $\tau, \varepsilon>0$, consider the functions $u$ and $v$ related by

$$
v(x)=\phi_{\varepsilon, 0}^{\tau}\left(\frac{\bar{x}}{\varepsilon}\right) u(x), \quad \text { for a.e. } x=\left(\bar{x}, x_{3}\right) \in \omega \times I .
$$

Then $v \in H_{0}^{1}(\omega \times I)$ if and only if $u \in H_{0}^{1}(\omega \times I)$. Moreover, if $v \in H_{0}^{1}(\omega \times I)$, then

$$
\int_{\omega \times I} \bar{A}_{\varepsilon}(\bar{x}) \bar{\nabla} v(x) \bar{\nabla} v(x)+\frac{a_{33}^{\varepsilon}(\bar{x})}{\varepsilon^{2 \tau}} \pi^{2}|v(x)|^{2}-\frac{\mu_{\varepsilon, 0}^{\tau}}{\varepsilon^{2 \tau}}|v(x)|^{2} \mathrm{~d} x=\int_{\omega \times I}\left[\phi_{\varepsilon, 0}^{\tau}\left(\frac{\bar{x}}{\varepsilon}\right)\right]^{2} \bar{A}_{\varepsilon}(\bar{x}) \bar{\nabla} u(x) \bar{\nabla} u(x) \mathrm{d} x .
$$


Proof. We proceed in two steps.

Step 1. We begin by proving that equality (2.4) holds for every $u \in H_{0}^{1}(\omega \times I) \cap L^{\infty}(\omega \times I)$.

Since $\phi_{\varepsilon, 0}^{\tau} \in H_{\#}^{1}(Y) \cap C_{\#}^{0, s}(Y)$, for some $0<s<1$, then for any $u \in H_{0}^{1}(\omega \times I) \cap L^{\infty}(\omega \times I)$ the function $v$ defined by $(2.3)$ also belongs to $H_{0}^{1}(\omega \times I) \cap L^{\infty}(\omega \times I)$. For $u \in C_{0}^{\infty}(\omega \times I)$ we have

$$
\begin{aligned}
\int_{\omega \times I} \bar{A}_{\varepsilon}(\bar{x}) & \bar{\nabla} v(x) \bar{\nabla} v(x)+\frac{a_{33}^{\varepsilon}(\bar{x})}{\varepsilon^{2 \tau}} \pi^{2}|v(x)|^{2}-\frac{\mu_{\varepsilon, 0}^{\tau}}{\varepsilon^{2 \tau}}|v(x)|^{2} \mathrm{~d} x \\
= & \int_{\omega \times I}-\phi_{\varepsilon, 0}^{\tau}\left(\frac{\bar{x}}{\varepsilon}\right) u(x) \operatorname{div}_{\bar{x}}\left\{\bar{A}_{\varepsilon}(\bar{x}) \bar{\nabla}\left[\phi_{\varepsilon, 0}^{\tau}\left(\frac{\bar{x}}{\varepsilon}\right) u(x)\right]\right\}+\left|\phi_{\varepsilon, 0}^{\tau}\left(\frac{\bar{x}}{\varepsilon}\right)\right|^{2}|u(x)|^{2} \frac{a_{33}^{\varepsilon}(\bar{x}) \pi^{2}-\mu_{\varepsilon, 0}^{\tau}}{\varepsilon^{2 \tau}} \mathrm{d} x
\end{aligned}
$$

where $\operatorname{div}_{\bar{x}}$ stands for the divergence in the variables $x_{1}, x_{2}$. Considering the definition of $\phi_{\varepsilon, 0}^{\tau}(\dot{\bar{\varepsilon}})$, it can be checked (see also [10]) that

$$
\begin{aligned}
-\phi_{\varepsilon, 0}^{\tau}\left(\frac{\bar{x}}{\varepsilon}\right) \operatorname{div}_{\bar{x}}\left\{\bar{A}_{\varepsilon}(\bar{x}) \bar{\nabla}\left[\phi_{\varepsilon, 0}^{\tau}\left(\frac{\bar{x}}{\varepsilon}\right) u(x)\right]\right\}+\left|\phi_{\varepsilon, 0}^{\tau}\left(\frac{\bar{x}}{\varepsilon}\right)\right|^{2} \frac{a_{33}^{\varepsilon}(\bar{x}) \pi^{2}-\mu_{\varepsilon, 0}^{\tau}}{\varepsilon^{2 \tau}} u(x) & =-\operatorname{div}_{\bar{x}}\left\{\left|\phi_{\varepsilon, 0}^{\tau}\left(\frac{\bar{x}}{\varepsilon}\right)\right|^{2} \overline{A_{\varepsilon}}(\bar{x}) \bar{\nabla} u(x)\right\}
\end{aligned}
$$

Combining this relation with (2.5) and integrating by parts in $\omega$ yields $(2.4)$ for all $u \in C_{0}^{\infty}(\omega \times I)$. In order to show that (2.4) also holds true for all $u \in H_{0}^{1}(\omega \times I) \cap L^{\infty}(\omega \times I)$, it suffices to approximate such a function $u$ by a sequence of $C_{0}^{\infty}(\omega \times I)$ functions and to pass to the limit in (2.4).

Step 2. In this step we prove that if $v \in H_{0}^{1}(\omega \times I)$, then the function $u$ given by $(2.3)$ belongs to $H_{0}^{1}(\omega \times I)$ and

$$
\int_{\omega \times I} \bar{A}_{\varepsilon}(\bar{x}) \bar{\nabla} v(x) \bar{\nabla} v(x)+\frac{a_{33}^{\varepsilon}(\bar{x})}{\varepsilon^{2 \tau}} \pi^{2}|v(x)|^{2}-\frac{\mu_{\varepsilon, 0}^{\tau}}{\varepsilon^{2 \tau}}|v(x)|^{2} \mathrm{~d} x \geq \int_{\omega \times I}\left|\phi_{\varepsilon, 0}^{\tau}\left(\frac{\bar{x}}{\varepsilon}\right)\right|^{2} \bar{A}_{\varepsilon}(\bar{x}) \bar{\nabla} u(x) \bar{\nabla} u(x) \mathrm{d} x .
$$

Let $v \in H_{0}^{1}(\omega \times I)$ be an arbitrary function. Since $\phi_{\varepsilon, 0}^{\tau} \in H_{\#}^{1}(Y) \cap C_{\#}^{0, s}(Y)$ is strictly positive, the function

$$
u(x):=\frac{v(x)}{\phi_{\varepsilon, 0}^{\tau}\left(\frac{\bar{x}}{\varepsilon}\right)}, \quad \text { a.e. } x=\left(\bar{x}, x_{3}\right) \in \omega \times I,
$$

is well defined and belongs to $L^{2}(\omega \times I)$. Moreover, $\nabla_{3} u \in L^{2}(\omega \times I)$.

Let $\left\{v_{n}\right\}_{n \in \mathbb{N}}$ be a sequence in $C_{0}^{\infty}(\omega \times I)$ such that $v_{n} \rightarrow v$ in $H_{0}^{1}(\omega \times I)$ as $n \rightarrow \infty$. Setting $u_{n}:=v_{n} / \phi_{\varepsilon, 0}^{\tau}$, we have $u_{n} \rightarrow u$ and $\nabla_{3} u_{n} \rightarrow \nabla_{3} u$ in $L^{2}(\omega \times I)$ as $n \rightarrow \infty$. Furthermore, for all $n \in \mathbb{N}, u_{n} \in H_{0}^{1}(\omega \times I) \cap L^{\infty}(\omega \times I)$, and so, by Step 1,

$$
\int_{\omega \times I} \bar{A}_{\varepsilon}(\bar{x}) \bar{\nabla} v_{n}(x) \bar{\nabla} v_{n}(x)+\frac{a_{33}^{\varepsilon}(\bar{x})}{\varepsilon^{2 \tau}} \pi^{2}\left|v_{n}(x)\right|^{2}-\frac{\mu_{\varepsilon, 0}^{\tau}}{\varepsilon^{2 \tau}}\left|v_{n}(x)\right|^{2} \mathrm{~d} x=\int_{\omega \times I}\left|\phi_{\varepsilon, 0}^{\tau}\left(\frac{\bar{x}}{\varepsilon}\right)\right|^{2} \bar{A}_{\varepsilon}(\bar{x}) \bar{\nabla} u_{n}(x) \bar{\nabla} u_{n}(x) \mathrm{d} x .
$$

The convergence $v_{n} \underset{n \rightarrow \infty}{\longrightarrow} v$ in $H_{0}^{1}(\omega \times I)$ yields

$$
\begin{aligned}
\lim _{n \rightarrow \infty} \int_{\omega \times I} \bar{A}_{\varepsilon}(\bar{x}) \bar{\nabla} v_{n}(x) \bar{\nabla} v_{n}(x)+\frac{a_{33}^{\varepsilon}(\bar{x})}{\varepsilon^{2 \tau}} \pi^{2}\left|v_{n}(x)\right|^{2}-\frac{\mu_{\varepsilon, 0}^{\tau}}{\varepsilon^{2 \tau}}\left|v_{n}(x)\right|^{2} \mathrm{~d} x & \\
& =\int_{\omega \times I} \bar{A}_{\varepsilon}(\bar{x}) \bar{\nabla} v(x) \bar{\nabla} v(x)+\frac{a_{33}^{\varepsilon}(\bar{x})}{\varepsilon^{2 \tau}} \pi^{2}|v(x)|^{2}-\frac{\mu_{\varepsilon, 0}^{\tau}}{\varepsilon^{2 \tau}}|v(x)|^{2} \mathrm{~d} x
\end{aligned}
$$


which, together with (2.7), implies that

$$
\sup _{n \in \mathbb{N}}\left\{\int_{\omega \times I}\left|\phi_{\varepsilon, 0}^{\tau}\left(\frac{\bar{x}}{\varepsilon}\right)\right|^{2} \bar{A}_{\varepsilon}(\bar{x}) \bar{\nabla} u_{n}(x) \bar{\nabla} u_{n}(x) \mathrm{d} x\right\}<+\infty .
$$

Consequently, since there is a constant $c_{\varepsilon}>0$ such that $\phi_{\varepsilon, 0}^{\tau}(\cdot / \varepsilon)>c_{\varepsilon}$, from (1.1) we get $\sup _{n}\left\|\bar{\nabla} u_{n}\right\|_{L^{2}\left(\omega \times I ; \mathbb{R}^{2}\right)}<$ $+\infty$. Therefore, $u \in H_{0}^{1}(\omega \times I)$ and $u_{n} \rightarrow u$ weakly in $H_{0}^{1}(\omega \times I)$ as $n \rightarrow \infty$.

Using the sequential lower semicontinuity with respect to the weak topology of $L^{2}\left(\omega \times I ; \mathbb{R}^{2}\right)$ of the convex functional $F: L^{2}\left(\omega \times I ; \mathbb{R}^{2}\right) \rightarrow \mathbb{R}$ defined by

$$
F(w):=\int_{\omega \times I}\left|\phi_{\varepsilon, 0}^{\tau}\left(\frac{\bar{x}}{\varepsilon}\right)\right|^{2} \bar{A}_{\varepsilon}(\bar{x}) w(x) w(x) \mathrm{d} x, \quad w \in L^{2}\left(\omega \times I ; \mathbb{R}^{2}\right),
$$

we conclude that

$$
\liminf _{n \rightarrow \infty} \int_{\omega \times I}\left|\phi_{\varepsilon, 0}^{\tau}\left(\frac{\bar{x}}{\varepsilon}\right)\right|^{2} \bar{A}_{\varepsilon}(\bar{x}) \bar{\nabla} u_{n}(x) \bar{\nabla} u_{n}(x) \mathrm{d} x \geq \int_{\omega \times I}\left|\phi_{\varepsilon, 0}^{\tau}\left(\frac{\bar{x}}{\varepsilon}\right)\right|^{2} \bar{A}_{\varepsilon}(\bar{x}) \bar{\nabla} u(x) \bar{\nabla} u(x) \mathrm{d} x .
$$

From (2.7)-(2.9) we deduce (2.6).

Changing the roles of $u$ and $v$ we conclude that if $u \in H_{0}^{1}(\omega \times I)$ then $v$ also belongs to $H_{0}^{1}(\omega \times I)$, and the converse of (2.6) holds true.

We now recall a classic result of homogenization (see [7], Thm. 13.12), which will be particularly useful in the cases $\varepsilon \approx \delta$ and $\varepsilon \ll \delta$; namely, to prove that a convenient sequence of functionals satisfies condition (iii) of Proposition 2.1.

Proposition 2.4. Let $B \in\left[L^{\infty}\left(\mathbb{R}^{2}\right)\right]^{2 \times 2}$ be a $2 \times 2$ real, symmetric and $Y$-periodic matrix satisfying bounds (1.1) with $0<\zeta<\eta$. For each $\varepsilon>0$, define $B_{\varepsilon}(\cdot):=B(\dot{\bar{\varepsilon}})$. Then, there exists a $2 \times 2$ constant matrix $B^{h}$ such that the sequence of functionals $\left\{J_{\varepsilon}\right\}_{\varepsilon>0}$, where $J_{\varepsilon}: H_{0}^{1}(\omega) \rightarrow \mathbb{R}$ is given by

$$
J_{\varepsilon}(\varphi):=\int_{\omega} B_{\varepsilon}(\bar{x}) \bar{\nabla} \varphi(\bar{x}) \bar{\nabla} \varphi(\bar{x}) \mathrm{d} \bar{x}
$$

$\Gamma$-converges as $\varepsilon \rightarrow 0^{+}$, with respect to the weak topology of $H_{0}^{1}(\omega)$, to the functional $J: H_{0}^{1}(\omega) \rightarrow \mathbb{R}$ defined by

$$
J(\varphi):=\int_{\omega} B^{h} \bar{\nabla} \varphi(\bar{x}) \bar{\nabla} \varphi(\bar{x}) \mathrm{d} \bar{x} .
$$

The matrix $B^{h}$ is called the homogenized limit of the sequence $\left\{B_{\varepsilon}\right\}_{\varepsilon>0}$.

Unfortunately, the lack of a positive uniform lower bound for $\left\{\phi_{\varepsilon, 0}^{\tau}\right\}_{\varepsilon>0}$ when $\tau>1$ will prevent us from using Proposition 2.4, and consequently Proposition 2.1, in the case $\varepsilon \gg \delta$. To treat this last case we will make use of an alternative result that shows that the spectrum $\sigma_{\varepsilon}^{\delta}$ associated with the tridimensional problem (1.5) equals a countable union of spectra associated with certain bidimensional problems.

Proposition 2.5. Let $B \in\left[L^{\infty}\left(\mathbb{R}^{2}\right)\right]^{2 \times 2}$ be a $2 \times 2$ real, symmetric and $Y$-periodic matrix satisfying bounds (1.1) with $0<\zeta<\eta$. Let $b \in L^{\infty}\left(\mathbb{R}^{2}\right)$ be a $Y$-periodic function such that $\zeta \leq b(\bar{y}) \leq \eta$ for a.e. $\bar{y} \in Y$. Given $n \in \mathbb{N}$, let $\lambda_{k}^{(n)}$ be the kth eigenvalue for the bidimensional spectral problem

$$
\left\{\begin{array}{l}
-\operatorname{div}\left(B(\bar{x}) \bar{\nabla} \varphi_{n}\right)+b(\bar{x}) \varsigma_{n} \varphi_{n}=\lambda_{n} \varphi_{n}, \text { a.e. } \bar{x} \in \omega, \\
\varphi_{n} \in H_{0}^{1}(\omega),
\end{array}\right.
$$


where, we recall, $\left(\varsigma_{n}, \theta_{n}\right)$ is the nth eigenpair for problem (1.7). Then $\left\{\lambda_{k}^{(n)}\right\}_{k, n \in \mathbb{N}}$ can be written as a nondecreasing sequence $\left\{\tilde{\lambda}_{m}\right\}_{m \in \mathbb{N}}$, where eigenvalues are repeated according to their multiplicity, which coincides with the spectral sequence of the tridimensional spectral problem

$$
\left\{\begin{array}{l}
-\operatorname{div}(B(\bar{x}) \bar{\nabla} v)-b(\bar{x}) \Delta_{3} v=\lambda v, \text { a.e. }\left(\bar{x}, x_{3}\right) \in \omega \times I, \\
v \in H_{0}^{1}(\omega \times I) .
\end{array}\right.
$$

In particular, $\lambda_{1}=\tilde{\lambda}_{1}=\lambda_{1}^{(1)}$.

Proof. Denote by $\left(\lambda_{k}^{(n)}, \varphi_{k}^{(n)}\right)$ a $L^{2}(\omega)$-normalized $k$ th eigenpair for problem (2.10). Then, it can be checked that:

(1) The family of functions $\left\{v_{k}^{(n)}=\varphi_{k}^{(n)}(\bar{x}) \theta_{n}\left(x_{3}\right), n=1,2, \ldots, k=1,2, \ldots\right\}$ is an orthonormal basis in $L^{2}(\omega \times I)$;

(2) $\left(\lambda_{k}^{(n)}, v_{k}^{(n)}\right), k, n \in \mathbb{N}$, are eigenpairs of $(2.11)$.

Since the operator $\left(-\operatorname{div}(B(\bar{x}) \bar{\nabla})-b(\bar{x}) \Delta_{3}\right)$, whose domain is a linear subset of $H_{0}^{1}(\omega \times I)$ dense in $L^{2}(\omega \times I)$, is a coercive self-adjoint operator in $L^{2}(\omega \times I)$ with a compact resolvent, in view of (1) and (2) and using the Fredholm Theorem, we conclude that all its eigenvalues belong to $\left\{\lambda_{k}^{(n)}\right\}_{k, n \in \mathbb{N}^{*}}$ This completes the proof.

\section{Proof of Theorem $1.1(\varepsilon \approx \delta)$}

In this section we prove Theorem 1.1. Let us recall that $\left(\mu_{0}, \phi_{0}\right)$ is the first $L^{2}(Y)$-normalized eigenpair for problem (1.6) with $\tau=1$, while $\left(\varsigma_{1}, \theta_{1}\right)=\left(\pi^{2}, \sqrt{2} \cos \left(\pi x_{3}\right)\right)$ is the first $L^{2}(I)$-normalized eigenpair for problem (1.7). Since we are expecting the asymptotic behavior mentioned in (1.8) for the shifted spectrum $\sigma_{\varepsilon}-\frac{\mu_{0}}{\varepsilon^{2}}$, instead of the energy defined in (1.4) for $\delta=\varepsilon$, we consider the functional $I_{\varepsilon}: L^{2}(\omega \times I) \rightarrow[0,+\infty]$, defined by

$$
I_{\varepsilon}(v):=\left\{\begin{array}{lc}
\int_{\omega \times I} \bar{A}_{\varepsilon}(\bar{x}) \bar{\nabla} v(x) \bar{\nabla} v(x)+\frac{a_{33}^{\varepsilon}(\bar{x})}{\varepsilon^{2}}\left|\nabla_{3} v(x)\right|^{2}-\frac{\mu_{0}}{\varepsilon^{2}}|v(x)|^{2} \mathrm{~d} x, & \text { if } v \in H_{0}^{1}(\omega \times I), \\
+\infty, & \text { otherwise. }
\end{array}\right.
$$

Using Proposition 2.3 with $\tau=1$, we conclude that $I_{\varepsilon}(v)=G_{\varepsilon}(u)$, where $G_{\varepsilon}: L^{2}(\omega \times I) \rightarrow[0,+\infty]$ is the functional given by

$$
G_{\varepsilon}(u):= \begin{cases}\int_{\omega \times I} \bar{B}_{\varepsilon}(\bar{x}) \bar{\nabla} u(x) \bar{\nabla} u(x)+\frac{b_{33}^{\varepsilon}(\bar{x})}{\varepsilon^{2}}\left(\left|\nabla_{3} u(x)\right|^{2}-\pi^{2}|u(x)|^{2}\right) \mathrm{d} x, & \text { if } u \in H_{0}^{1}(\omega \times I), \\ +\infty, & \text { otherwise },\end{cases}
$$

where, a.e. $\bar{x} \in \omega$,

$$
\bar{B}_{\varepsilon}(\bar{x}):=\left(b_{\alpha \beta}^{\varepsilon}(\bar{x})\right) \in \mathbb{M}^{2 \times 2}, \quad b_{\alpha \beta}^{\varepsilon}(\bar{x}):=\left[\phi_{0}\left(\frac{\bar{x}}{\varepsilon}\right)\right]^{2} a_{\alpha \beta}\left(\frac{\bar{x}}{\varepsilon}\right), \quad b_{33}^{\varepsilon}(\bar{x}):=\left[\phi_{0}\left(\frac{\bar{x}}{\varepsilon}\right)\right]^{2} a_{33}\left(\frac{\bar{x}}{\varepsilon}\right) .
$$

Remark 3.1. Notice that since $\phi_{0} \in H_{\#}^{1}(Y) \cap C_{\#}^{0, s}(Y)$, for some $0<s<1$, is strictly positive, we have that $B_{\varepsilon}:=\left(\phi_{0}(\dot{\bar{\varepsilon}})\right)^{2} A_{\varepsilon}$ is a $3 \times 3$ real, symmetric and $\varepsilon Y$-periodic matrix, satisfying a condition of the type (1.1).

In order to prove Theorem 1.1, we must check that the sequence $\left\{G_{\varepsilon}\right\}_{\varepsilon>0}$ satisfies the hypotheses of Proposition 2.1. 
Proposition 3.2. Let $G_{\varepsilon}$ be the functional in (3.2). Then the sequence $\left\{G_{\varepsilon}\right\}_{\varepsilon>0} \Gamma$-converges, with respect to the strong topology of $L^{2}(\omega \times I)$, to the functional $G: L^{2}(\omega \times I) \rightarrow[0,+\infty]$ defined by

$$
G(u):= \begin{cases}\int_{\omega} \bar{B}^{h} \bar{\nabla} \varphi(\bar{x}) \bar{\nabla} \varphi(\bar{x}) \mathrm{d} \bar{x}, & \text { if } u\left(\bar{x}, x_{3}\right)=\varphi(\bar{x}) \theta_{1}\left(x_{3}\right), \varphi \in H_{0}^{1}(\omega), \\ +\infty, & \text { otherwise, }\end{cases}
$$

where the constant matrix $\bar{B}^{h}$ is the homogenized limit of the sequence $\left\{\bar{B}_{\varepsilon}\right\}_{\varepsilon>0}$. Moreover, $G_{\varepsilon}$ also satisfies conditions (i) and (ii) in Proposition 2.1.

Proof. We will proceed in two steps.

Step 1. We prove that if $u_{\varepsilon}, u \in L^{2}(\omega \times I)$ are such that $u_{\varepsilon} \rightarrow u$ in $L^{2}(\omega \times I)$ as $\varepsilon \rightarrow 0^{+}$, then $G(u) \leq$ $\liminf _{\varepsilon \rightarrow 0^{+}} G_{\varepsilon}\left(u_{\varepsilon}\right)$. Furthermore, conditions (i) and (ii) in Proposition 2.1 are satisfied.

We start by observing that if $w \in H_{0}^{1}(\omega \times I)$, then for a.e. $\bar{x} \in \omega, w(\bar{x}, \cdot) \in H_{0}^{1}(I)$. Thus, since $\varsigma_{1}=\pi^{2}$ is the first eigenvalue associated with problem (1.7), we have, a.e. $\bar{x} \in \omega$,

$$
\int_{I}\left(\left|\nabla_{3} w\right|^{2}-\pi^{2}|w|^{2}\right) \mathrm{d} x_{3} \geq 0
$$

The uniform ellipticity of $B_{\varepsilon}$ (see Rem. 3.1) and (3.3) ensure that $G_{\varepsilon} \geq 0$ in $L^{2}(\omega \times I)$. Hence, condition (i) in Proposition 2.1 is satisfied.

Let $u_{\varepsilon}, u \in L^{2}(\omega \times I)$ be as in the statement of Step 1. Up to a subsequence (which we will not relabel), we may assume without loss of generality that

$$
\liminf _{\varepsilon \rightarrow 0^{+}} G_{\varepsilon}\left(u_{\varepsilon}\right)=\lim _{\varepsilon \rightarrow 0^{+}} G_{\varepsilon}\left(u_{\varepsilon}\right)<+\infty .
$$

Then $\left\{u_{\varepsilon}\right\}_{\varepsilon>0} \subset H_{0}^{1}(\omega \times I)$ and $\sup _{\varepsilon} G_{\varepsilon}\left(u_{\varepsilon}\right)<+\infty$. Using (3.3), the uniform ellipticity of $B_{\varepsilon}$ and the uniform bound of $\left\{u_{\varepsilon}\right\}_{\varepsilon>0}$ in $L^{2}(\omega \times I)$, we get

$$
\int_{\omega \times I}\left|\bar{\nabla} u_{\varepsilon}\right|^{2} \mathrm{~d} x \leq C, \quad \int_{\omega \times I}\left|\nabla_{3} u_{\varepsilon}\right|^{2} \mathrm{~d} x \leq C \varepsilon^{2}+\pi^{2} \int_{\omega \times I}\left|u_{\varepsilon}\right|^{2} \mathrm{~d} x \leq \bar{C},
$$

where $C$ and $\bar{C}$ are constants independent of $\varepsilon$. Consequently, $\sup _{\varepsilon}\left\|u_{\varepsilon}\right\|_{H_{0}^{1}(\omega \times I)}<+\infty$ and $u_{\varepsilon} \rightarrow u$ weakly in $H_{0}^{1}(\omega \times I)$. The sequential lower semicontinuity of the $L^{2}$-norm with respect to the weak topology and (3.4) yield

$$
\int_{\omega \times I}\left(\left|\nabla_{3} u\right|^{2}-\pi^{2}|u|^{2}\right) \mathrm{d} x \leq 0 .
$$

Hence, taking into account (3.3), $\int_{I}\left(\left|\nabla_{3} u\right|^{2}-\pi^{2}|u|^{2}\right) \mathrm{d} x_{3}=0$, a.e. $\bar{x} \in \omega$, from which we deduce that there is a function $\varphi \in H_{0}^{1}(\omega)$ such that $u\left(\bar{x}, x_{3}\right)=\varphi(\bar{x}) \theta_{1}\left(x_{3}\right)$, a.e. $\left(\bar{x}, x_{3}\right) \in \omega \times I$.

Using Fubini's theorem, Fatou's Lemma, Proposition 2.4 (see also Rem. 3.1) and the condition $\left\|\theta_{1}\right\|_{L^{2}(I)}=1$, we obtain

$$
\liminf _{\varepsilon \rightarrow 0^{+}} G_{\varepsilon}\left(u_{\varepsilon}\right) \geq \liminf _{\varepsilon \rightarrow 0^{+}} \int_{\omega \times I} \bar{B}_{\varepsilon}(\bar{x}) \bar{\nabla} u_{\varepsilon}(x) \bar{\nabla} u_{\varepsilon}(x) \mathrm{d} x \geq \int_{I}\left[\int_{\omega} \bar{B}^{h} \bar{\nabla} u(x) \bar{\nabla} u(x) \mathrm{d} \bar{x}\right] \mathrm{d} x_{3}=G(u) .
$$

Finally, to conclude Step 1, we observe that if $\sup _{\varepsilon} G_{\varepsilon}\left(u_{\varepsilon}\right)<+\infty$ and $\sup _{\varepsilon}\left\|u_{\varepsilon}\right\|_{L^{2}(\omega \times I)}<+\infty$, then (3.4) holds. Consequently, condition (ii) in Proposition 2.1 is also satisfied.

Step 2. We prove that for any $u \in L^{2}(\omega \times I)$, there exists a sequence $\left\{u_{\varepsilon}\right\}_{\varepsilon>0} \subset L^{2}(\omega \times I)$ satisfying $u_{\varepsilon} \rightarrow u$ in $L^{2}(\omega \times I)$ as $\varepsilon \rightarrow 0^{+}$, and $G(u)=\lim _{\varepsilon \rightarrow 0^{+}} G_{\varepsilon}\left(u_{\varepsilon}\right)$. 
Given $u \in L^{2}(\omega \times I)$, the only nontrivial case is when $u\left(\bar{x}, x_{3}\right)=\varphi(\bar{x}) \theta_{1}\left(x_{3}\right)$, with $\varphi \in H_{0}^{1}(\omega)$, otherwise, considering Step 1, it's enough to take $u_{\varepsilon} \equiv u$.

By Proposition 2.4, there exists a sequence $\left\{\varphi_{\varepsilon}\right\}_{\varepsilon>0} \subset H_{0}^{1}(\omega)$ converging in $L^{2}(\omega)$ to $\varphi$ and such that

$$
\lim _{\varepsilon \rightarrow 0^{+}} \int_{\omega} \bar{B}_{\varepsilon}(\bar{x}) \bar{\nabla} \varphi_{\varepsilon}(\bar{x}) \bar{\nabla} \varphi_{\varepsilon}(\bar{x}) \mathrm{d} \bar{x}=\int_{\omega} \bar{B}^{h} \bar{\nabla} \varphi(\bar{x}) \bar{\nabla} \varphi(\bar{x}) \mathrm{d} \bar{x} .
$$

Recalling that $\int_{I}\left(\left|\theta_{1}^{\prime}\right|^{2}-\pi^{2}\left|\theta_{1}\right|^{2}\right) \mathrm{d} x_{3}=0$, in order to obtain the intended equality it suffices to define $u_{\varepsilon}\left(\bar{x}, x_{3}\right):=$ $\varphi_{\varepsilon}(\bar{x}) \theta_{1}\left(x_{3}\right)$. This concludes Step 2 as well as the proof of Proposition 3.2.

We are now in position to prove Theorem 1.1.

Proof of Theorem 1.1. Let $H_{\varepsilon}$ be the Hilbert space $H:=L^{2}(\omega \times I)$ endowed with the scalar product $(\cdot \mid \cdot)_{\varepsilon}$, where

$$
(u \mid v)_{\varepsilon}:=\int_{\omega \times I}\left[\phi_{0}\left(\frac{\bar{x}}{\varepsilon}\right)\right]^{2} u(x) v(x) \mathrm{d} x, \quad u, v \in L^{2}(\omega \times I) .
$$

Since $\phi_{0} \in H_{\#}^{1}(Y) \cap C_{\#}^{0, s}(Y)$ is a strictly positive function, there exist $0<c_{1}<c_{2}$ such that for all $\bar{y} \in Y$, $c_{1}<\phi_{0}(\bar{y})<c_{2}$. Moreover, by Riemann-Lebesgue's Lemma,

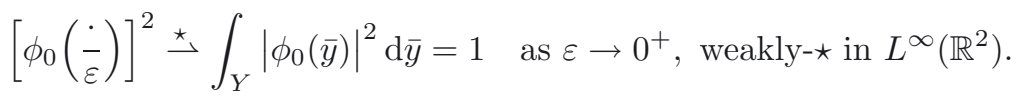

Hence conditions (2.1) and (2.2) hold. On the other hand, for each $\varepsilon>0, G_{\varepsilon}$ defined in (3.2) is a nonnegative lower semicontinuous quadratic form in $L^{2}(\omega \times I)$. Consequently, the associated operator, $\mathcal{A}_{\varepsilon}$, is a self-adjoint operator in $H_{\varepsilon}$ (see [7], Thm. 12.13). Let $\left\{\left(\nu_{\varepsilon, k}, u_{\varepsilon, k}\right)\right\}_{k \in \mathbb{N}}$ and $\left\{\left(\nu_{k}, \varphi_{k}\right)\right\}_{k \in \mathbb{N}}$ be such that

$$
\begin{array}{llll}
u_{\varepsilon, k} \in H_{0}^{1}(\omega \times I), & \mathcal{A}_{\varepsilon} u_{\varepsilon, k}=\nu_{\varepsilon, k} u_{\varepsilon, k}, & \nu_{\varepsilon, 1} \leq \nu_{\varepsilon, 2} \leq \cdots \leq \nu_{\varepsilon, k} \leq \cdots, & \left(u_{\varepsilon, k} \mid u_{\varepsilon, l}\right)_{\varepsilon}=\delta_{k l}, \\
\varphi_{k} \in H_{0}^{1}(\omega), & -\operatorname{div}_{\bar{x}}\left(\bar{B}^{h} \bar{\nabla} \varphi_{k}\right)=\nu_{k} \varphi_{k}, & \nu_{1} \leq \nu_{2} \leq \cdots \leq \nu_{k} \leq \cdots, & \left(\varphi_{k} \mid \varphi_{l}\right)=\delta_{k l},
\end{array}
$$

where $(\cdot \cdot \cdot)$ represents the standard scalar product in $L^{2}(\omega)$.

By Propositions 2.1 and 3.2, $\nu_{\varepsilon, k} \rightarrow \nu_{k}$ as $\varepsilon \rightarrow 0^{+}$. Moreover, up to a subsequence that we do not relabel, $u_{\varepsilon, k} \rightarrow u_{k}$ weakly in $H_{0}^{1}(\omega \times I)$ as $\varepsilon \rightarrow 0^{+}$, where $u_{k}$ is the product between an eigenfunction associated with $\nu_{k}$ and $\theta_{1}$. Conversely, any eigenfunction $u_{k}=\varphi_{k} \theta_{1}$ is the weak limit of a particular sequence of eigenfunctions associated with $\nu_{\varepsilon, k}$.

To finish the proof of Theorem 1.1 we are left to show that (1.8) holds. Considering for each $k \in \mathbb{N}, \mu_{k} \in \mathbb{R}$ and functions $w_{k}$ and $\tilde{w}_{k}$ such that

$$
w_{k}(x)=\phi_{0}\left(\frac{\bar{x}}{\varepsilon}\right) \tilde{w}_{k}(x), \quad \text { a.e. } x=\left(\bar{x}, x_{3}\right) \in \omega \times I,
$$

Proposition 2.3 implies that $w_{k}$ belongs to $H_{0}^{1}(\omega \times I)$ if, and only if, $\tilde{w}_{k}$ belongs to $H_{0}^{1}(\omega \times I)$, and also that the equalities

$$
G_{\varepsilon}\left(\tilde{w}_{k}\right)=\left(\mathcal{A}_{\varepsilon} \tilde{w}_{k} \mid \tilde{w}_{k}\right)_{\varepsilon}=\mu_{k}\left(\tilde{w}_{k} \mid \tilde{w}_{k}\right)_{\varepsilon}, \quad\left(\tilde{w}_{k} \mid \tilde{w}_{l}\right)_{\varepsilon}=\delta_{k l}
$$

hold true if, and only if, the equalities

$$
I_{\varepsilon}\left(w_{k}\right)=\left(-\operatorname{div}_{\bar{x}}\left(\bar{A}_{\varepsilon} \bar{\nabla} w_{k}\right)-\frac{a_{33}^{\varepsilon}}{\varepsilon^{2}} \Delta_{3} w_{k}-\frac{\mu_{0}}{\varepsilon^{2}} w_{k} \mid w_{k}\right)=\mu_{k}\left(w_{k} \mid w_{k}\right), \quad\left(w_{k} \mid w_{l}\right)_{\varepsilon}=\delta_{k l}
$$

are satisfied, where $(\cdot \mid \cdot)$ represents the standard scalar product in $L^{2}(\omega \times I)$ and $I_{\varepsilon}$ is the functional in (3.1). Replacing $\mu_{k}$ by $\nu_{\varepsilon, k}, w_{k}$ by $v_{\varepsilon, k}$ and $\tilde{w}_{k}$ by $u_{\varepsilon, k}$, we conclude the proof of (1.8). 


\section{Proof of Theorem $1.2(\varepsilon \ll \delta)$}

This section is devoted to the proof of Theorem 1.2. The arguments are similar to those of Theorem 1.1, however, in this case problem (1.6) does depend on $\varepsilon$, this compels us to study the asymptotic behavior of its first $L^{2}(Y)$-normalized eigenpair $\left(\mu_{\varepsilon, 0}^{\tau}, \phi_{\varepsilon, 0}^{\tau}\right)$ as $\varepsilon \rightarrow 0^{+}$. Throughout this section we assume that $\tau \in(0,1)$ is fixed, and that $\delta=\varepsilon^{\tau}$.

Proposition 4.1. Assume that, in addition to the hypotheses made in the beginning of Section 1 , a $a_{\alpha \beta}$ are uniformly Lipschitz continuous in $Y$. Let $\left\{\left(\varrho_{j}, \psi_{j}\right)\right\}_{j \in \mathbb{N}_{0}}$ be given by (1.9)-(1.10), and let $i \in \mathbb{N}$ be such that $\frac{i-1}{i}<\tau \leq \frac{i}{i+1}$. Then $\phi_{\varepsilon, 0}^{\tau}(\cdot / \varepsilon) \rightarrow 1 \equiv \psi_{0}$ uniformly in $\omega$, and $\mu_{\varepsilon, 0}^{\tau}$ behaves as follows:

$$
\mu_{\varepsilon, 0}^{\tau}=\varrho_{0}+\varepsilon^{2(1-\tau)} \varrho_{1}+\cdots+\varepsilon^{2 i(1-\tau)} \varrho_{i}+o\left(\varepsilon^{2 i(1-\tau)}\right)
$$

Proof. Let us start by proving that $\mu_{\varepsilon, 0}^{\tau} \rightarrow \varrho_{0}=\pi^{2} \int_{Y} a_{33}(\bar{y}) \mathrm{d} \bar{y}>0$, and that all the others eigenvalues of problem (1.6) tend to $+\infty$ as $\varepsilon \rightarrow 0^{+}$. By Rayleigh's formula for $\mu_{\varepsilon, 0}^{\tau}$,

$$
\mu_{\varepsilon, 0}^{\tau}=\min _{\substack{\phi \in H_{\#}^{1}(Y) \\\|\phi\|_{L^{2}(Y)}\left({ }^{2}\right.}}\left\{\int_{Y} \frac{1}{\varepsilon^{2(1-\tau)}} \bar{A}(\bar{y}) \bar{\nabla} \phi(\bar{y}) \bar{\nabla} \phi(\bar{y})+a_{33}(\bar{y}) \pi^{2}|\phi(\bar{y})|^{2} \mathrm{~d} \bar{y}\right\}
$$

Using (1.1) and $\phi \equiv 1$ as a test function in (4.2), we conclude that $\zeta \pi^{2} \leq \mu_{\varepsilon, 0}^{\tau} \leq \varrho_{0}$. In particular, $\lim \sup _{\varepsilon \rightarrow 0^{+}} \mu_{\varepsilon, 0}^{\tau} \leq \varrho_{0}$. Since $\phi_{\varepsilon, 0}^{\tau}$ is a minimizer for $\mu_{\varepsilon, 0}^{\tau}$, using again (1.1) we deduce that $\left\|\bar{\nabla} \phi_{\varepsilon, 0}^{\tau}\right\|_{L^{2}(Y)} \rightarrow 0$. Consequently, $\phi_{\varepsilon, 0}^{\tau} \rightarrow 1$ in $H_{\#}^{1}(Y)$. In turn, this implies $\liminf \operatorname{in\rightarrow 0}_{\varepsilon \rightarrow 0^{+}} \mu_{\varepsilon, 0}^{\tau} \geq \liminf \operatorname{lin}_{\varepsilon \rightarrow 0^{+}} \int_{Y} a_{33}(\bar{y}) \pi^{2}\left|\phi_{\varepsilon, 0}^{\tau}(\bar{y})\right|^{2} \mathrm{~d} \bar{y}=$ $\varrho_{0}$. Therefore, $\mu_{\varepsilon, 0}^{\tau} \rightarrow \varrho_{0}$ as $\varepsilon \rightarrow 0^{+}$.

Similarly, using Rayleigh's formula for $\mu_{\varepsilon, 1}$ and admitting that the latter is bounded, we are led to a contradiction, since we would conclude that any minimizing sequence of eigenfunctions must converge on the one hand to the constant function $\psi_{0} \equiv 1$ and on the other hand to a function having zero mean (by the orthogonality condition). So, except for the first, all the eigenvalues of problem (1.6) tend to $+\infty$ as $\varepsilon \rightarrow 0^{+}$.

We now prove the statement on the asymptotic behavior of $\phi_{\varepsilon, 0}^{\tau}$. If, in addition, we suppose that $a_{\alpha \beta}$ are uniformly Lipschitz continuous in $Y$, then (see, [9], Thm. 8.8) $\left\{\phi_{\varepsilon, 0}^{\tau}\right\}_{\varepsilon>0}$ is uniformly bounded in $H^{2}(Y)$. Due to the compact injection of $H^{2}(Y)$ in $C^{0}(\bar{Y})$, we conclude that $\phi_{\varepsilon, 0}^{\tau}(\bar{y}) \rightarrow 1$ uniformly in $\bar{Y}$ as $\varepsilon \rightarrow 0^{+}$. Finally, the $Y$-periodicity of $\phi_{\varepsilon, 0}^{\tau}$ ensures that $\phi_{\varepsilon, 0}^{\tau}(\cdot / \varepsilon) \rightarrow 1$ uniformly in $\omega$ as $\varepsilon \rightarrow 0^{+}$.

We are left to establish (4.1). It is based on the Vishik-Lyusternik Lemma (see [16,18]): Let $L: H \rightarrow H$ be a linear compact self-adjoint operator in a Hilbert space $H$, and suppose that $\lambda>0$ and $f \in H$ are such that $\|L f-\lambda f\|_{H} \leq \gamma$, for some constant $\gamma \in \mathbb{R}^{+}$. Then there exists an eigenvalue $\bar{\lambda}$ of $L$ such that $|\bar{\lambda}-\lambda| \leq \gamma\|f\|_{H}^{-1}$.

For the sake of simplicity we will present the proof only for $i=1$, the argument being easily generalized for $i>1$. Considering $H=L_{\#}^{2}(Y)$, and setting $\epsilon:=\varepsilon^{2(1-\tau)}, \mu_{\epsilon}:=\mu_{\varepsilon, 0}^{\tau} b(\bar{y}):=\pi^{2} a_{33}(\bar{y})$ and $\mathcal{A}_{\epsilon} \varphi:=$ $-\frac{1}{\epsilon} \operatorname{div}(\bar{A}(\bar{y}) \bar{\nabla} \varphi)+b(\bar{y}) \varphi, \varphi \in H_{\#}^{1}(Y)$, we apply the above result to $L_{\epsilon}: L_{\#}^{2}(Y) \rightarrow L_{\#}^{2}(Y)$ such that $L_{\epsilon} g=\varphi$, solution of $\mathcal{A}_{\epsilon} \varphi=g$, to $f_{\epsilon}:=\mathcal{A}_{\epsilon} \psi_{\epsilon}, \psi_{\epsilon}:=\psi_{0}+\epsilon \psi_{1}+\epsilon^{2} \psi_{2}$, and to $\lambda_{\epsilon}=\left(\varrho_{0}+\epsilon \varrho_{1}\right)^{-1}$.

Since $L_{\epsilon} f_{\epsilon}-\lambda_{\epsilon} f_{\epsilon}=\psi_{\epsilon}-\lambda_{\epsilon} \mathcal{A}_{\epsilon} \psi_{\epsilon}=: w_{\epsilon}$, using (1.9)-(1.10) we conclude that

$$
w_{\epsilon}=\left(\epsilon^{2}\left(\left(b-\varrho_{0}\right) \psi_{2}-\varrho_{1} \psi_{1}\right)-\epsilon^{3} \varrho_{1} \psi_{2}\right)\left(\varrho_{0}+\epsilon \varrho_{1}\right)^{-1}
$$

In view of the condition $\varrho_{0}>0$, we deduce that for all $\epsilon>0$ small enough and for a constant $c$ independent of $\epsilon,\left\|w_{\epsilon}\right\|_{H} \leq \epsilon^{2} c$. Consequently, there exists an eigenvalue $\bar{\lambda}_{\epsilon}$ satisfying $\left|\bar{\lambda}_{\epsilon}-\left(\varrho_{0}+\epsilon \varrho_{1}\right)^{-1}\right| \leq \epsilon^{2} c$, for some other constant $c$ independent of $\epsilon$, where we used the fact that $\left\|\mathcal{A}_{\epsilon} \psi_{\epsilon}\right\|_{H} \rightarrow \varrho_{0}>0$. Since all the eigenvalues of $L_{\epsilon}$ tend to zero, except for the first, which converges to $\varrho_{0}^{-1}>0$, we conclude that for all $\epsilon$ small enough, $\bar{\lambda}_{\epsilon}=\mu_{\epsilon}^{-1}$. Hence, $\left|\mu_{\epsilon}-\left(\varrho_{0}+\epsilon \varrho_{1}\right)\right| \leq \epsilon^{2} c$, for some other constant $c$ independent of $\epsilon$. This concludes the proof for $i=1$. 
As it was already mentioned, the main ideas of the proof of Theorem 1.2 are those of Theorem 1.1. We are expecting the asymptotic behavior referred in (1.12) for the shifted spectrum $\sigma_{\varepsilon}-\frac{\mu_{\varepsilon}^{\tau}, 0}{\varepsilon^{2 \tau}}$ (see also (4.1)), and so instead of the energy defined in (1.4) for $\delta=\varepsilon^{\tau}$, we consider the functional $I_{\varepsilon}^{\tau}: L^{2}(\omega \times I) \rightarrow[0,+\infty]$, defined by

$$
I_{\varepsilon}^{\tau}(v):= \begin{cases}\int_{\omega \times I} \bar{A}_{\varepsilon}(\bar{x}) \bar{\nabla} v(x) \bar{\nabla} v(x)+\frac{a_{33}^{\varepsilon}(\bar{x})}{\varepsilon^{2 \tau}}\left|\nabla_{3} v(x)\right|^{2}-\frac{\mu_{\varepsilon, 0}^{\tau}}{\varepsilon^{2 \tau}}|v(x)|^{2} \mathrm{~d} x, & \text { if } v \in H_{0}^{1}(\omega \times I), \\ +\infty, & \text { otherwise. }\end{cases}
$$

By Proposition 2.3, we have that $I_{\varepsilon}^{\tau}(v)=G_{\varepsilon}^{\tau}(u)$, where $G_{\varepsilon}^{\tau}: L^{2}(\omega \times I) \rightarrow[0,+\infty]$ is the functional given by

$$
G_{\varepsilon}^{\tau}(u):=\left\{\begin{array}{lc}
\int_{\omega \times I} \bar{B}_{\varepsilon}^{\tau}(\bar{x}) \bar{\nabla} u(x) \bar{\nabla} u(x)+\frac{b_{33}^{\tau, \varepsilon}(\bar{x})}{\varepsilon^{2}}\left(\left|\nabla_{3} u(x)\right|^{2}-\pi^{2}|u(x)|^{2}\right) \mathrm{d} x, & \text { if } u \in H_{0}^{1}(\omega \times I), \\
+\infty, & \text { otherwise },
\end{array}\right.
$$

and, a.e. $\bar{x} \in \omega$,

$$
\bar{B}_{\varepsilon}^{\tau}(\bar{x}):=\left(b_{\alpha \beta}^{\tau, \varepsilon}(\bar{x})\right) \in \mathbb{M}^{2 \times 2}, \quad b_{\alpha \beta}^{\tau, \varepsilon}(\bar{x}):=\left[\phi_{\varepsilon, 0}^{\tau}\left(\frac{\bar{x}}{\varepsilon}\right)\right]^{2} a_{\alpha \beta}\left(\frac{\bar{x}}{\varepsilon}\right), \quad b_{33}^{\tau, \varepsilon}(\bar{x}):=\left[\phi_{\varepsilon, 0}^{\tau}\left(\frac{\bar{x}}{\varepsilon}\right)\right]^{2} a_{33}\left(\frac{\bar{x}}{\varepsilon}\right) .
$$

The analogue to Proposition 3.2 reads as follows.

Proposition 4.2. Let $G_{\varepsilon}^{\tau}$ be the functional in (4.3). Then the sequence $\left\{G_{\varepsilon}^{\tau}\right\}_{\varepsilon>0} \Gamma$-converges, with respect to the strong topology of $L^{2}(\omega \times I)$, to the functional $G^{\tau}: L^{2}(\omega \times I) \rightarrow[0,+\infty]$ defined by

$$
G^{\tau}(u):= \begin{cases}\int_{\omega} \bar{A}^{h} \bar{\nabla} \varphi(\bar{x}) \bar{\nabla} \varphi(\bar{x}) \mathrm{d} \bar{x}, & \text { if } u\left(\bar{x}, x_{3}\right)=\varphi(\bar{x}) \theta_{1}\left(x_{3}\right), \varphi \in H_{0}^{1}(\omega), \\ +\infty, & \text { otherwise }\end{cases}
$$

where $\bar{A}^{h}$ is the homogenized limit of the sequence $\left\{\bar{A}_{\varepsilon}\right\}_{\varepsilon>0}$. Moreover, $G_{\varepsilon}^{\tau}$ also satisfies conditions (i) and (ii) in Proposition 2.1.

Proof. The proof is very similar to that of Proposition 3.2, however we outline the main differences.

Step 1. We prove that if $u_{\varepsilon}, u \in L^{2}(\omega \times I)$ are such that $u_{\varepsilon} \rightarrow u$ in $L^{2}(\omega \times I)$ as $\varepsilon \rightarrow 0^{+}$, then $G^{\tau}(u) \leq$ $\liminf _{\varepsilon \rightarrow 0^{+}} G_{\varepsilon}^{\tau}\left(u_{\varepsilon}\right)$. Furthermore, conditions (i) and (ii) in Proposition 2.1 are satisfied.

Without loss of generality we may assume that $\liminf _{\varepsilon \rightarrow 0^{+}} G_{\varepsilon}^{\tau}\left(u_{\varepsilon}\right)=\lim _{\varepsilon \rightarrow 0^{+}} G_{\varepsilon}^{\tau}\left(u_{\varepsilon}\right)<+\infty$. Then, using (1.1) and the uniform convergence $\phi_{\varepsilon, 0}^{\tau}(\cdot / \varepsilon) \rightarrow 1$ in $\omega$ (see Prop. 4.1), we conclude that (3.4) holds. Consequently, $u_{\varepsilon} \rightarrow u$ weakly in $H_{0}^{1}(\omega \times I)$ as $\varepsilon \rightarrow 0^{+}$, where $u\left(\bar{x}, x_{3}\right)=\varphi(\bar{x}) \theta_{1}\left(x_{3}\right)$ for some $\varphi \in H_{0}^{1}(\omega)$, a.e. $\left(\bar{x}, x_{3}\right) \in \omega \times I$.

Fix $0<\gamma<1$. Then for all $\varepsilon$ sufficiently small, $\left[\phi_{\varepsilon, 0}^{\tau}(\cdot / \varepsilon)\right]^{2} \geq 1-\gamma$. Therefore, Fubini's Theorem, (1.1), Fatou's Lemma, Proposition 2.4 and the condition $\left\|\theta_{1}\right\|_{L^{2}(I)}=1$ ensure that

$$
\begin{aligned}
\liminf _{\varepsilon \rightarrow 0^{+}} G_{\varepsilon}^{\tau}\left(u_{\varepsilon}\right) & \geq \liminf _{\varepsilon \rightarrow 0^{+}} \int_{\omega \times I} \bar{B}_{\varepsilon}^{\tau}(\bar{x}) \bar{\nabla} u_{\varepsilon}(x) \bar{\nabla} u_{\varepsilon}(x) \mathrm{d} x \\
& \geq(1-\gamma) \int_{I}\left[\liminf _{\varepsilon \rightarrow 0^{+}} \int_{\omega} \bar{A}_{\varepsilon}(\bar{x}) \bar{\nabla} u_{\varepsilon}(x) \bar{\nabla} u_{\varepsilon}(x) \mathrm{d} \bar{x}\right] \mathrm{d} x_{3} \geq(1-\gamma) G^{\tau}(u),
\end{aligned}
$$

from which we conclude that $G^{\tau}(u) \leq \liminf _{\varepsilon \rightarrow 0^{+}} G_{\varepsilon}^{\tau}\left(u_{\varepsilon}\right)$ by letting $\gamma \rightarrow 0^{+}$.

To prove that $G_{\varepsilon}^{\tau}$ satisfies conditions (i) and (ii) in Proposition 2.1 it suffices to repeat the correspondent arguments in Step 1 of Proposition 3.2. This concludes Step 1. 
Step 2. We prove that for any $u \in L^{2}(\omega \times I)$, there exists a sequence $\left\{u_{\varepsilon}\right\}_{\varepsilon>0} \subset L^{2}(\omega \times I)$ satisfying $u_{\varepsilon} \rightarrow u$ in $L^{2}(\omega \times I)$ as $\varepsilon \rightarrow 0^{+}$, and $G^{\tau}(u)=\lim _{\varepsilon \rightarrow 0^{+}} G_{\varepsilon}^{\tau}\left(u_{\varepsilon}\right)$.

Given $u \in L^{2}(\omega \times I)$, the only nontrivial case is when $u\left(\bar{x}, x_{3}\right)=\varphi(\bar{x}) \theta_{1}\left(x_{3}\right)$, with $\varphi \in H_{0}^{1}(\omega)$, otherwise, considering Step 1, it's enough to take $u_{\varepsilon} \equiv u$.

By Proposition 2.4, there exists a sequence $\left\{\varphi_{\varepsilon}\right\}_{\varepsilon>0} \subset H_{0}^{1}(\omega)$ converging in $L^{2}(\omega)$ to $\varphi$ and such that

$$
\lim _{\varepsilon \rightarrow 0^{+}} \int_{\omega} \bar{A}_{\varepsilon}(\bar{x}) \bar{\nabla} \varphi_{\varepsilon}(\bar{x}) \bar{\nabla} \varphi_{\varepsilon}(\bar{x}) \mathrm{d} \bar{x}=\int_{\omega} \bar{A}^{h} \bar{\nabla} \varphi(\bar{x}) \bar{\nabla} \varphi(\bar{x}) \mathrm{d} \bar{x} .
$$

Fix $\gamma>0$. Let $\varepsilon_{0}>0$ be such that for all $0<\varepsilon \leq \varepsilon_{0},\left[\phi_{\varepsilon, 0}^{\tau}(\cdot / \varepsilon)\right]^{2} \leq 1+\gamma$. Define $u_{\varepsilon}\left(\bar{x}, x_{3}\right):=\varphi_{\varepsilon}(\bar{x}) \theta_{1}\left(x_{3}\right)$. Recalling that $\int_{I}\left(\left|\theta_{1}^{\prime}\right|^{2}-\pi^{2}\left|\theta_{1}\right|^{2}\right) \mathrm{d} x_{3}=0$, from (4.4) and (1.1) we conclude that

$$
\begin{aligned}
\limsup _{\varepsilon \rightarrow 0^{+}} G_{\varepsilon}^{\tau}\left(u_{\varepsilon}\right) & =\limsup _{\varepsilon \rightarrow 0^{+}} \int_{\omega} \bar{B}_{\varepsilon}^{\tau}(\bar{x}) \bar{\nabla} \varphi_{\varepsilon}(x) \bar{\nabla} \varphi_{\varepsilon}(x) \mathrm{d} x \\
& \leq(1+\gamma) \int_{\omega} \bar{A}^{h} \bar{\nabla} \varphi(\bar{x}) \bar{\nabla} \varphi(\bar{x}) \mathrm{d} \bar{x}=(1+\gamma) G^{\tau}(u) .
\end{aligned}
$$

Letting $\gamma \rightarrow 0^{+}$and using Step 1, we conclude Step 2 as well as the proof of Proposition 4.2.

Proof of Theorem 1.2. Replacing $G_{\varepsilon}$ by $G_{\varepsilon}^{\tau},\left(\mu_{0}, \phi_{0}\right)$ by $\left(\mu_{\varepsilon, 0}^{\tau}, \psi_{\varepsilon, 0}^{\tau}\right)$, and recalling Propositions 4.1 and 4.2 , the proof of Theorem 1.2 is analogous to that of Theorem 1.1.

\section{Proof of Theorems 1.4 And $1.8(\varepsilon \gg \delta)$}

Throughout this section we assume that $\tau \in(1,+\infty)$ is fixed, and that $\delta=\varepsilon^{\tau}$. As we mentioned before, the lack of a positive uniform lower bound for $\left\{\phi_{\varepsilon, 0}^{\tau}\right\}_{\varepsilon>0}$ will prevent us from using Proposition 2.1. So, in order to prove Theorems 1.4 and 1.8, we will take advantage essentially of Propositions 2.3 and 2.5, and of the asymptotic behavior of the eigenpair $\left(\mu_{\varepsilon, 0}^{\tau}, \phi_{\varepsilon, 0}^{\tau}\right)$ introduced in (1.6), which is the aim of the following lemmas.

To simplify the statements and the proof of the lemmas, we introduce some notations: $b:=\left(a_{33}-a_{\min }\right) \pi^{2}$, $\epsilon:=\varepsilon^{\tau-1}, \mu_{\epsilon}:=\frac{\mu_{\varepsilon, 0}^{\tau}-a_{\min } \pi^{2}}{\varepsilon^{2(\tau-1)}}, \phi_{\epsilon}:=\phi_{\varepsilon, 0}^{\tau}$. Problem (1.6) then reads

$$
-\operatorname{div}\left(\bar{A} \bar{\nabla} \phi_{\epsilon}\right)+\frac{b}{\epsilon^{2}} \phi_{\epsilon}=\mu_{\epsilon} \phi_{\epsilon}, \quad \phi_{\epsilon} \in H_{\#}^{1}(Y), \quad\left\|\phi_{\epsilon}\right\|_{L^{2}(Y)}=1
$$

The asymptotic behavior of $\left(\mu_{\epsilon}, \phi_{\epsilon}\right)$ depends strongly on the behavior of the potential $b$. As we referred in the Introduction, an interesting case is when $b$ oscillates between two different values and this justifies the present hypotheses on the coefficients.

Lemma 5.1. Under the hypotheses of Theorem 1.4 and using the above notations, let $\left(\nu_{0}, q_{0}\right)$ represent the $L^{2}(Q)$-normalized first eigenpair for problem (1.13), and consider $q_{0}$ extended by zero to the whole $Y$. Let also $\mu_{\epsilon, 1}$ represent the second eigenvalue for problem (5.1) and $\nu_{1}$ the second eigenvalue for problem (1.13). Then $\left\{\phi_{\epsilon}\right\}_{\epsilon>0}$ converges in norm to $q_{0}$ in $L^{2}(Y)$ and weakly in $H^{1}(Y)$. Moreover,

$$
\mu_{\epsilon} \rightarrow \nu_{0}, \quad \mu_{\epsilon, 1} \rightarrow \nu_{1} \quad \text { as } \epsilon \rightarrow 0^{+} .
$$

Proof. We will only prove the first convergence in (5.2), since the second one is analogous having in mind that the orthogonality condition $\int_{Y} \phi q_{0} \mathrm{~d} \bar{y}=0$ reads $\int_{Q} \phi q_{0} \mathrm{~d} \bar{y}=0$. 
Step 1. We prove that $\mu_{\epsilon} \leq \nu_{0}$.

Noticing that $b$ vanishes in $Q$, the eigenvalue $\mu_{\epsilon}$ is given by the Rayleigh's formula

$$
\mu_{\epsilon}=\inf _{\substack{\phi \in H_{\#}^{1}(Y) \\\|\phi\|_{L^{2}(Y)}=1}}\left\{\int_{Y} \bar{A} \bar{\nabla} \phi \bar{\nabla} \phi \mathrm{d} \bar{y}+\frac{1}{\epsilon^{2}} \int_{Y \backslash Q} b|\phi|^{2} \mathrm{~d} \bar{y}\right\} .
$$

Using in (5.3) test functions $q \in H_{0}^{1}(Q)$, with $\|q\|_{L^{2}(Q)}=1$, extended by zero to the whole $Y$, we obtain

$$
\mu_{\epsilon} \leq \inf _{\substack{q \in H_{0}^{1}(Q) \\\|q\|_{L^{2}(Q)}=1}}\left\{\int_{Q} \bar{A} \bar{\nabla} q \bar{\nabla} q \mathrm{~d} \bar{y}\right\}=\nu_{0},
$$

which concludes Step 1.

Step 2. We establish the convergence of $\left\{\phi_{\epsilon}\right\}_{\epsilon>0}$.

In the previous step we proved that

$$
\mu_{\epsilon}=\int_{Y} \bar{A} \bar{\nabla} \phi_{\epsilon} \bar{\nabla} \phi_{\epsilon} \mathrm{d} \bar{y}+\frac{1}{\epsilon^{2}} \int_{Y \backslash Q} b\left|\phi_{\epsilon}\right|^{2} \mathrm{~d} \bar{y} \leq \nu_{0}=\int_{Q} \bar{A} \bar{\nabla} q_{0} \bar{\nabla} q_{0} \mathrm{~d} \bar{y} .
$$

Consequently,

$$
\int_{Y} \bar{A} \bar{\nabla} \phi_{\epsilon} \bar{\nabla} \phi_{\epsilon} \mathrm{d} \bar{y} \leq \int_{Q} \bar{A} \bar{\nabla} q_{0} \bar{\nabla} q_{0} \mathrm{~d} \bar{y}, \quad \int_{Y \backslash Q} b\left|\phi_{\epsilon}\right|^{2} \mathrm{~d} \bar{y} \leq \epsilon^{2} \nu_{0}
$$

Using (1.1), from the first estimate in (5.6) we conclude that $\left\|\nabla \phi_{\epsilon}\right\|_{L^{2}(Y)}$ is bounded independently of $\epsilon$. Hence, up to a subsequence, $\left\{\phi_{\epsilon}\right\}_{\epsilon>0}$ converges to some $\phi_{0} \in H_{\#}^{1}(Y)$ weakly in $H^{1}(Y)$ and strongly in $L^{2}(Y)$. A lower semicontinuity argument then yields

$$
\int_{Q} \bar{A} \bar{\nabla} \phi_{0} \bar{\nabla} \phi_{0} \mathrm{~d} \bar{y} \leq \int_{Y} \bar{A} \bar{\nabla} \phi_{0} \bar{\nabla} \phi_{0} \mathrm{~d} \bar{y} \leq \liminf _{\epsilon \rightarrow 0+} \int_{Y} \bar{A} \bar{\nabla} \phi_{\epsilon} \bar{\nabla} \phi_{\epsilon} \mathrm{d} \bar{y} \leq \int_{Q} \bar{A} \bar{\nabla} q_{0} \bar{\nabla} q_{0} \mathrm{~d} \bar{y} .
$$

Fix $c>0$ such that $b(\cdot) \geq c$ on $Y \backslash Q$. Then, in view of the second estimate in (5.6),

$$
\left\|\phi_{\epsilon}\right\|_{L^{2}(Y \backslash \bar{Q})}^{2}=\int_{Y \backslash \bar{Q}}\left|\phi_{\epsilon}\right|^{2} \mathrm{~d} \bar{y} \leq \frac{\epsilon^{2}}{c} \nu_{0} \underset{\epsilon \rightarrow 0^{+}}{\longrightarrow} 0 .
$$

Thus $\phi_{0}=0$ a.e. in $Y \backslash \bar{Q}$. Consequently, $\phi_{0} \in H_{0}^{1}(Q)$ and $\left\|\phi_{0}\right\|_{L^{2}(Q)}=1$. Finally, from (5.7) and since $\phi_{0}$ is admissible in the variational definition of $\nu_{0}$, we obtain $\phi_{0} \equiv q_{0}$, as well as the convergence of the whole sequence $\left\{\phi_{\epsilon}\right\}_{\epsilon>0}$.

Step 3. We prove that $\mu_{\epsilon} \rightarrow \nu_{0}$ as $\epsilon \rightarrow 0^{+}$.

By (5.5), we have

$$
\mu_{\epsilon}=\int_{Y} \bar{A} \bar{\nabla} \phi_{\epsilon} \bar{\nabla} \phi_{\epsilon} \mathrm{d} \bar{y}+\frac{1}{\epsilon^{2}} \int_{Y \backslash Q} b\left|\phi_{\epsilon}\right|^{2} \mathrm{~d} \bar{y} \geq \int_{Y} \bar{A} \bar{\nabla} \phi_{\epsilon} \bar{\nabla} \phi_{\epsilon} \mathrm{d} \bar{y},
$$

and so, in view of (5.7) and since $\phi_{0} \equiv q_{0}$,

$$
\liminf _{\epsilon \rightarrow 0^{+}} \mu_{\epsilon} \geq \int_{Q} \bar{A} \bar{\nabla} q_{0} \bar{\nabla} q_{0} \mathrm{~d} \bar{y}=\nu_{0},
$$

which, together with (5.4), concludes Step 3. 
Lemma 5.2. Under the hypotheses of Theorem 1.4 and using the previous notations, the $L^{2}(Y)$-normalized first eigenpair $\left(\mu_{\epsilon}, \phi_{\epsilon}\right)$ for problem (5.1) has the following asymptotic behavior for any integer $n \in \mathbb{N}$ :

$$
\mu_{\epsilon}=\nu_{0}+\epsilon \mu_{1}+\epsilon^{2} \mu_{2}+\cdots+\epsilon^{n} \mu_{n}+\rho_{n, \epsilon}
$$

where $\mu_{i}, i \in\{1, \ldots, n\}$, are well determined constants and $\left|\rho_{n, \epsilon}\right| \leq c_{n} \epsilon^{n+\frac{1}{2}}$, for some positive constant $c_{n}$ independent of $\epsilon$, and

$$
\phi_{\epsilon}=q_{0}+\epsilon \phi_{1, \epsilon}+\epsilon^{2} \phi_{2, \epsilon}+\cdots+\epsilon^{n} \phi_{n, \epsilon}+r_{n, \epsilon},
$$

where $\phi_{i, \epsilon}, i \in\{1, \ldots, n\}$, are well-defined functions in $L^{2}(Y)$ and $\left\|r_{n, \epsilon}\right\|_{L^{2}(Y)} \leq \bar{c}_{n} \epsilon^{n+\frac{1}{2}}$ for a certain positive constant $\bar{c}_{n}$ independent of $\epsilon$.

Proof. The proof is based on the asymptotic expansion technique. We will detail the proof for $n=1$, being clear how to extend it for the higher orders.

For $\gamma>0$ we define $Q_{\gamma}:=\{\bar{y} \in Y: \operatorname{dist}(\bar{y}, Q)<\gamma\}$. Let $\gamma_{0}>0$ be such that the outward normals to $\partial Q$ of length $2 \gamma_{0}$ do not intersect. Consider a system of local coordinates $(s, \theta)$ on $Q_{2 \gamma_{0}} \backslash Q$, where $\theta$ represents the local coordinate on $\partial Q$ and $s \in\left[0,2 \gamma_{0}\right)$ stands for the distance to $\partial Q$ in the outward normal direction. In this local coordinates, equation (5.1) in $Q_{2 \gamma_{0}} \backslash \bar{Q}$ reads

$$
-\operatorname{div}\left(\bar{A}^{\star} \bar{\nabla} \varphi_{\epsilon}\right)+b^{\star} \cdot \bar{\nabla} \varphi_{\epsilon}+\frac{b}{\epsilon^{2}} \varphi_{\epsilon}=\mu_{\epsilon} \varphi_{\epsilon}
$$

for a certain uniformly elliptic matrix $\bar{A}^{\star}=\left(a_{\alpha \beta}^{\star}\right)$ with smooth coefficients as functions of $s$ and $\theta$, and for a certain vector $b^{\star}=\left(b_{1}^{\star}, b_{2}^{\star}\right)$, where $b_{1}^{\star}, b_{2}^{\star}$ are also smooth functions of $s$ and $\theta$.

In the sequel we will deal with different coordinates on different sides of $\partial Q$. For the sake of simplicity we will abusively identify $f(\bar{y})$ with $f(\bar{y}(s, \theta))$ or, conversely, $g(s, \theta)$ with $g(s(\bar{y}), \theta(\bar{y}))$.

For small $\epsilon>0$ we search for $\phi_{\epsilon}$ and $\mu_{\epsilon}$ with the following development

$$
\begin{aligned}
& \mu_{\epsilon}=\nu_{0}+\epsilon \mu_{1}+\epsilon^{2} \mu_{2}+\cdots, \\
& \phi_{\epsilon}=q_{0}+\epsilon \phi_{1, \epsilon}+\epsilon^{2} \phi_{2, \epsilon}+\cdots,
\end{aligned}
$$

where, we recall, $\left(\nu_{0}, q_{0}\right)$ is the $L^{2}(Q)$-normalized first eigenpair for problem (1.13), and $\phi_{i, \epsilon}, i \geq 1$, have the form

$$
\phi_{i, \epsilon}(\bar{y}):= \begin{cases}\phi_{i}^{-}(\bar{y}), & \text { in } Q, \\ \phi_{i}^{+}\left(\frac{s}{\epsilon}, \theta\right), & \text { in } Q_{2 \gamma_{0}} \backslash Q, \\ 0, & \text { in } Y \backslash Q_{2 \gamma_{0}} .\end{cases}
$$

In view of the regularity assumptions on the coefficients $a_{\alpha \beta}$ and $b$ and on the domain $Q$, the following Taylor expansions for $\theta$ fixed hold true

$$
\begin{aligned}
a_{\alpha \beta}^{\star}(s, \theta) & =a_{\alpha \beta}^{\star}(0, \theta)+\frac{\partial a_{\alpha \beta}^{\star}}{\partial s}(0, \theta) s+\frac{\partial^{2} a_{\alpha \beta}^{\star}}{\partial s^{2}}(0, \theta) \frac{s^{2}}{2}+\frac{\partial^{3} a_{\alpha \beta}^{\star}}{\partial s^{3}}(\xi(s), \theta) \frac{s^{3}}{3 !}, \\
b(s, \theta) & =b(0, \theta)+\frac{\partial b}{\partial s}(0, \theta) s+\frac{\partial^{2} b}{\partial s^{2}}(0, \theta) \frac{s^{2}}{2}+\frac{\partial^{3} b}{\partial s^{3}}(\eta(s), \theta) \frac{s^{3}}{3 !} .
\end{aligned}
$$

Setting $\tau=s / \epsilon, \tau \in\left[0,2 \gamma_{0} / \epsilon\right)$, substituting (5.9) and (5.10)-(5.11) in (5.8), using expressions (5.12)-(5.13) and collecting like powers of $\epsilon$, we obtain in $Q_{2 \gamma_{0}} \backslash \bar{Q}$, for the power $\epsilon^{-1}$, that $\phi_{1}^{+}$must satisfy

$$
-a_{11}^{\star}(0, \theta) \frac{\partial^{2} \phi_{1}^{+}}{\partial \tau^{2}}+b(0, \theta) \phi_{1}^{+}=0,
$$

where $\theta$ is a parameter. 
Denote by $\psi_{1}^{+}$the solution, for fixed $\theta$, of

Then

$$
\left\{\begin{array}{l}
-a_{11}^{\star}(0, \theta) \frac{\partial^{2} \psi_{1}^{+}}{\partial \tau^{2}}+b(0, \theta) \psi_{1}^{+}=0 \\
\lim _{\tau \rightarrow \infty} \psi_{1}^{+}(\tau, \theta)=0, \quad \frac{\partial \psi_{1}^{+}}{\partial \tau}(0, \theta)=-\frac{1}{a_{11}^{\star}(0, \theta)}
\end{array}\right.
$$

and we define

$$
\psi_{1}^{+}(\tau, \theta)=\frac{1}{\sqrt{a_{11}^{\star}(0, \theta) b(0, \theta)}} \mathrm{e}^{-\sqrt{\frac{b(0, \theta)}{a_{11}^{\star}(0, \theta)}} \tau},
$$

$$
\phi_{1}^{+}(\tau, \theta):=-\left[\left(\bar{A} \bar{\nabla} q_{0} n_{Q}\right)(\bar{y}(0, \theta))\right] \psi_{1}^{+}(\tau, \theta),
$$

where $n_{Q}$ represents the outward normal to $\partial Q$ at $\bar{y}(0, \theta)$, so that we may have

$$
\left(a_{11}^{\star} \frac{\partial \phi_{1}^{+}}{\partial \tau}\right)(0, \theta)=\left(\bar{A} \bar{\nabla} q_{0} n_{Q}\right)(\bar{y}(0, \theta)) .
$$

Also, $\phi_{1}^{-}$must satisfy

$$
\left\{\begin{array}{l}
-\operatorname{div}\left(\bar{A} \bar{\nabla} \phi_{1}^{-}\right)=\nu_{0} \phi_{1}^{-}+\mu_{1} q_{0}, \text { a.e. in } Q, \\
\phi_{1 \mid \partial Q}^{-}(\bar{y}(0, \theta))=\phi_{1}^{+}(0, \theta),
\end{array}\right.
$$

and, from the compatibility condition

$$
\int_{\partial Q}\left(\bar{A} \bar{\nabla} q_{0} n_{Q}\right) \phi_{1}^{+} \mathrm{d} \sigma=\mu_{1} \int_{Q}\left|q_{0}\right|^{2} \mathrm{~d} \bar{y}
$$

we obtain

$$
\mu_{1}=\int_{\partial Q}\left(\bar{A} \bar{\nabla} q_{0} n_{Q}\right) \phi_{1}^{+} \mathrm{d} \sigma=-\int_{\partial Q}\left|\bar{A} \bar{\nabla} q_{0} n_{Q}\right|^{2} \psi_{1}^{+} \mathrm{d} \sigma<0 .
$$

So, $\phi_{1}^{-}$is uniquely defined as the solution of (5.14) with $\mu_{1}$ given by (5.15), and satisfying

$$
\int_{Q} q_{0} \phi_{1}^{-} \mathrm{d} \bar{y}=0
$$

Collecting the terms of order $\epsilon^{0}$ we obtain in $Q_{2 \gamma_{0}} \backslash \bar{Q}$ that $\phi_{2}^{+}$must satisfy

$$
-a_{11}^{\star}(0, \theta) \frac{\partial^{2} \phi_{2}^{+}}{\partial \tau^{2}}+b(0, \theta) \phi_{2}^{+}=R(\tau, \theta)
$$

where $R(\tau, \theta)$ is a finite sum of functions of the type $f(\theta) \tau \mathrm{e}^{-c(\theta) \tau}$ with $f$ and $c>0$ being bounded functions of $\theta$. Therefore, problem

$$
\left\{\begin{array}{l}
-a_{11}^{\star}(0, \theta) \frac{\partial^{2} \phi_{2}^{+}}{\partial \tau^{2}}+b(0, \theta) \varphi=R(\tau, \theta), \\
\lim _{\tau \rightarrow \infty} \phi_{2}^{+}(\tau, \theta)=0, \quad a_{11}^{\star}(0, \theta) \frac{\partial \phi_{2}^{+}}{\partial \tau}(0, \theta)=\left(\bar{A} \bar{\nabla} \phi_{1}^{-} n_{Q}\right)(\bar{y}(0, \theta)),
\end{array}\right.
$$

has a unique solution $\phi_{2}^{+}$, which is smooth in $(\tau, \theta)$ and decays exponentially as $\tau \rightarrow \infty$.

We now define $\phi_{2}^{-}$in $Q$ as the solution in $Q$ of

$$
\left\{\begin{array}{l}
-\operatorname{div}\left(\bar{A} \bar{\nabla} \phi_{2}^{-}\right)=\nu_{0} \phi_{2}^{-}+\mu_{1} \phi_{1}^{-}+\mu_{2} q_{0}, \text { a.e. in } Q, \\
\phi_{2 \mid \partial Q}^{-}(\bar{y}(0, \theta))=\phi_{2}^{+}(0, \theta)
\end{array}\right.
$$


with

$$
\mu_{2}:=\int_{\partial Q}\left(\bar{A} \bar{\nabla} q_{0} n_{Q}\right) \phi_{2}^{+} \mathrm{d} \sigma
$$

so that the compatibility condition is satisfied.

Now, in order to make the function $\phi_{\epsilon}$ and its derivatives continuous at $\partial Q$, we introduce a smooth function $\psi_{2}^{-}$, defined on $Q$, such that $\psi_{2}^{-}{ }_{\mid \partial Q}=0, \bar{A} \bar{\nabla} \psi_{2}^{-} n_{Q}=-\bar{A} \bar{\nabla} \phi_{2}^{-} n_{Q}$. Consider also a cut-off function $\phi_{\gamma_{0}} \in$ $C^{\infty}(\mathbb{R} ;[0,1])$ such that $\phi_{\gamma_{0}}(s)=1$, if $s \leq \gamma_{0}$, and $\phi_{\gamma_{0}}(s)=0$, if $s \geq 2 \gamma_{0}$.

Finally, we set

$$
w_{\epsilon}(\bar{y}):= \begin{cases}q_{0}(\bar{y})+\epsilon \phi_{1}^{-}(\bar{y})+\epsilon^{2} \phi_{2}^{-}(\bar{y})+\epsilon^{2} \psi_{2}^{-}(\bar{y}), & \text { if } \bar{y} \in Q, \\ \left(\epsilon \phi_{1}^{+}\left(\frac{s(\bar{y})}{\epsilon}, \theta(\bar{y})\right)+\epsilon^{2} \phi_{2}^{+}\left(\frac{s(\bar{y})}{\epsilon}, \theta(\bar{y})\right)\right) \phi_{2 \gamma_{0}}(s(\bar{y})), & \text { if } \bar{y} \in Q_{2 \gamma_{0}} \backslash Q, \\ 0, & \text { if } \bar{y} \in Y \backslash Q_{2 \gamma_{0}},\end{cases}
$$

and

$$
\Lambda_{\epsilon}:=\nu_{0}+\epsilon \mu_{1}+\epsilon^{2} \mu_{2} .
$$

Then, it can be checked that for suitable constants $c_{0}$ and $c_{1}$ independent of $\epsilon$, the following estimates hold true

$$
\begin{aligned}
& \left\|w_{\epsilon}\right\|_{L^{2}(Y)} \leq 1+c_{0} \epsilon^{2}, \\
& \left\|-\operatorname{div}\left(\bar{A} \bar{\nabla} w_{\epsilon}\right)+\frac{1}{\epsilon^{2}} b w_{\epsilon}-\Lambda_{\epsilon} w_{\epsilon}\right\|_{L^{2}(Y)} \leq c_{1} \epsilon^{3 / 2} .
\end{aligned}
$$

Indeed, from (5.16) and the fact that $q_{0}$ vanishes outside $Q$ it follows that $\phi_{1, \epsilon}$ is orthogonal to $q_{0}$. Thus, considering the normalization $\left\|q_{0}\right\|_{L^{2}(Y)}=1$, we obtain

$$
\left\|q_{0}+\epsilon \phi_{1, \epsilon}\right\|_{L^{2}(Y)}^{2}=1+\epsilon^{2}\left\|\phi_{1, \epsilon}\right\|_{L^{2}(Y)}^{2} .
$$

This implies in particular that $\left\|q_{0}+\epsilon \phi_{1, \epsilon}\right\|_{L^{2}(Y)} \geq 1$ and $\left\|q_{0}+\epsilon \phi_{1, \epsilon}\right\|_{L^{2}(Y)} \leq\left\|q_{0}+\epsilon \phi_{1, \epsilon}\right\|_{L^{2}(Y)}^{2}$. Therefore, (5.19) is a consequence of $(5.21)$.

To justify (5.20), we use (5.17) and (5.18) and the definitions of all functions $q_{0}, \phi_{1}^{ \pm}, \phi_{2}^{ \pm}, \psi_{2}^{-}$and $\phi_{\gamma_{0}}$. After straightforward rearrangements we obtain

$$
-\operatorname{div}\left(\bar{A} \bar{\nabla} w_{\epsilon}\right)+\frac{1}{\epsilon^{2}} b w_{\epsilon}-\Lambda_{\epsilon} w_{\epsilon}= \begin{cases}\epsilon^{2} r_{\epsilon}^{-}(\bar{y}), & \bar{y} \in Q, \\ \epsilon r_{\epsilon}^{+}\left(s(\bar{y}), \theta(\bar{y}), \frac{s(\bar{y})}{\epsilon}\right), & \text { if } \bar{y} \in Q_{2 \gamma_{0} \backslash Q,} \backslash \\ 0, & \text { if } \bar{y} \in Y \backslash Q_{2 \gamma_{0}},\end{cases}
$$

where

$$
\left\|r_{\epsilon}^{-}\right\|_{L^{2}(Q)} \leq c_{2} \text { and }\left|r_{\epsilon}^{+}(s, \theta, \tau)\right| \leq c_{3} \tau^{j} \mathrm{e}^{-c_{4} \tau}
$$

with positive constants $c_{2}, c_{3}, c_{4}$, and $j \in \mathbb{N}$, independents of $\epsilon$. It follows from the second upper bound in (5.23) that for some positive constant $c_{5}$,

$$
\left\|r_{\epsilon}^{+}\right\|_{L^{2}(Y \backslash Q)}^{2} \leq c_{5} \epsilon .
$$

Then, in view of the first upper bound in (5.23) and thanks to (5.24) and (5.22), we obtain estimate (5.20).

In order to obtain the announced estimates we notice that, by Lemma 5.1, we can find $\epsilon_{0}>0$ such that for all $\epsilon<\epsilon_{0}$ the ground state $\mu_{\epsilon}$ and the second eigenvalue $\mu_{\epsilon, 1}$ of problem (5.1) satisfy the inequality $\mu_{\epsilon}-\mu_{\epsilon, 1} \geq \bar{c}>0$. So, using Vishik-Lyusternik Lemma (see $[16,18]$ ), from (5.19) and (5.20) we get

$$
\left|\Lambda_{\epsilon}-\mu_{\epsilon}\right| \leq c_{6} \epsilon^{3 / 2}, \quad\left\|w_{\epsilon}-\phi_{\epsilon}\right\|_{L^{2}(Y)} \leq c_{7} \epsilon^{3 / 2},
$$


for some positive constants $c_{6}, c_{7}$ independent of $\epsilon$. Considering the definitions of $w_{\epsilon}$ and $\Lambda_{\epsilon}$ we conclude, from (5.25), that

$$
\left|\mu_{\epsilon}-\left(\nu_{0}+\epsilon \mu_{1}\right)\right| \leq c_{8} \epsilon^{3 / 2}, \quad\left\|\phi_{\epsilon}-\left(q_{0}+\epsilon \phi_{1, \epsilon}\right)\right\|_{L^{2}(Y)} \leq c_{9} \epsilon^{3 / 2}
$$

for some constants $c_{8}>0$ and $c_{9}>0$. This completes the proof for $n=1$.

Remark 5.3. If the potential $b$ is constant in $Q$, continuous in $Y$ and has linear or quadratic growth in the vicinity of $\partial Q$, i.e. $b(\bar{y})=\alpha(\bar{y}) d(\bar{y})$ or $b(\bar{y})=\alpha(\bar{y}) d^{2}(\bar{y})$, for $\bar{y} \in Q_{\gamma_{0}} \backslash Q$, where $\alpha(\cdot)>0$ and $d(\cdot)=\operatorname{dist}(\cdot, \partial Q)$, the techniques are similar to the case treated in Lemma 5.2. In the linear growth case we obtain, for $n=1$, $\mu_{\epsilon}=\nu_{0}+\epsilon^{2 / 3} \mu_{1}$, and in the case of quadratic growth, also for $n=1, \mu_{\epsilon}=\nu_{0}+\epsilon^{1 / 2} \mu_{1}$.

We now prove Theorems 1.4 and 1.8 .

Proof of Theorem 1.4. By Proposition 2.5, the first eigenvalue $\lambda_{\varepsilon, 1}$ of the tridimensional problem (1.5) coincides with the first eigenvalue, $\lambda_{\varepsilon, 1}^{(1)}$, of the bidimensional problem in $H_{0}^{1}(\omega)$,

$$
-\operatorname{div}\left(\bar{A}_{\varepsilon}(\bar{x}) \bar{\nabla} \varphi_{\varepsilon}^{(1)}\right)+\frac{a_{33}^{\varepsilon}(\bar{x})}{\varepsilon^{2 \tau}} \pi^{2} \varphi_{\varepsilon}^{(1)}=\lambda_{\varepsilon}^{(1)} \varphi_{\varepsilon}^{(1)}, \quad \text { a.e. } \bar{x} \in \omega
$$

Also, the correspondent $L^{2}$-normalized eigenfunctions $v_{\varepsilon, 1}$ and $\varphi_{\varepsilon, 1}^{(1)}$ satisfy the following relation

$$
v_{\varepsilon, 1}(x)=\varphi_{\varepsilon, 1}^{(1)}(\bar{x}) \theta_{1}\left(x_{3}\right), \quad \text { a.e. } x=\left(\bar{x}, x_{3}\right) \in \omega \times I,
$$

where $\theta_{1}$ is the first $L^{2}(I)$-normalized eigenfunction of problem (1.7).

On the other hand, recalling the proof of Proposition 2.3, relation (2.4) holds true if we restrict (2.3) to $v$ and $u$ only depending on $\bar{x}$. Using (2.4), for $\varphi, \psi \in H_{0}^{1}(\omega)$ satisfying

$$
\varphi(\bar{x})=\phi_{\varepsilon, 0}^{\tau}\left(\frac{\bar{x}}{\varepsilon}\right) \psi(\bar{x}), \quad \text { a.e. } \bar{x} \in \omega
$$

we obtain

$$
\begin{aligned}
\lambda_{\varepsilon, 1} & =\lambda_{\varepsilon, 1}^{(1)}=\inf _{\substack{\varphi \in H_{0}^{1}(\omega) \\
\varphi \neq 0}} \frac{\int_{\omega} \bar{A}_{\varepsilon}(\bar{x}) \bar{\nabla} \varphi(\bar{x}) \bar{\nabla} \varphi(\bar{x})+\frac{a_{33}^{\varepsilon}(\bar{x})}{\varepsilon^{2 \tau}} \pi^{2}|\varphi(\bar{x})|^{2} \mathrm{~d} \bar{x}}{\int_{\omega}|\varphi(\bar{x})|^{2} \mathrm{~d} \bar{x}}, \\
& =\frac{\mu_{\varepsilon, 0}^{\tau}}{\varepsilon^{2 \tau}}+\inf _{\substack{\psi \in H_{0}^{1}(\omega) \\
\psi \neq 0}} \frac{\int_{\omega}\left[\phi_{\varepsilon, 0}^{\tau}\left(\frac{\bar{x}}{\varepsilon}\right)\right]^{2} \overline{A_{\varepsilon}}(\bar{x}) \bar{\nabla} \psi(\bar{x}) \bar{\nabla} \psi(\bar{x}) \mathrm{d} \bar{x}}{\int_{\omega}\left[\phi_{\varepsilon, 0}^{\tau}\left(\frac{\bar{x}}{\varepsilon}\right)\right]^{2}|\psi(\bar{x})|^{2} \mathrm{~d} \bar{x}} \\
& =\frac{\mu_{\varepsilon, 0}^{\tau}}{\varepsilon^{2 \tau}}+\nu_{\varepsilon, 1}^{\tau} .
\end{aligned}
$$

Using Lemma 5.1 and recalling the notations introduced at the beginning of this section, we get $\mu_{\varepsilon, 0}^{\tau} \rightarrow \nu_{0}$ and $\phi_{\varepsilon, 0}^{\tau} \rightarrow q_{0}$ weakly in $H_{\#}^{1}(Y)$ as $\varepsilon \rightarrow 0^{+}$, and

$$
\lambda_{\varepsilon, 1}=\frac{a_{\min } \pi^{2}}{\varepsilon^{2 \tau}}+\frac{\nu_{0}}{\varepsilon^{2}}+\varepsilon^{\tau-3} \mu_{1}+\cdots+\varepsilon^{k(\tau-1)-2} \mu_{k}+\rho_{\varepsilon}^{\tau}+\nu_{\varepsilon, 1}^{\tau}
$$

where $\left|\rho_{\varepsilon}^{\tau}\right| \leq C \varepsilon^{\left(k+\frac{1}{2}\right) \tau-\left(k+\frac{5}{2}\right)} \rightarrow 0$ as $\varepsilon \rightarrow 0^{+}$. 
To conclude the proof of Theorem 1.4 we are left to prove that $\nu_{\varepsilon, 1}^{\tau} \rightarrow 0$ as $\varepsilon \rightarrow 0^{+}$. Construct a sequence $\left\{\psi_{\varepsilon}\right\}_{\varepsilon>0}$ in $H_{0}^{1}(\omega)$ as follows: for each $\varepsilon>0$ let $K_{\varepsilon}:=\left\{\kappa \in \mathbb{Z}^{2}: \varepsilon(\kappa+Y) \subset \omega\right\}$, and define

$$
T_{\varepsilon}:=\operatorname{int}\left(\bigcup_{\kappa \in K_{\varepsilon}} \varepsilon\left(\kappa_{i}+\bar{Y}\right)\right) .
$$

Consider the cut-off function $\phi_{\gamma_{0}}$ introduced in Lemma 5.2 in the definition of $w_{\epsilon}$ (see (5.17)). Extend $\phi_{\gamma_{0}}$ to the whole $\mathbb{R}^{2}$ by $Y$-periodicity, and define $\psi_{\varepsilon}$ by $\psi_{\varepsilon}(\bar{x}):=\phi_{\gamma_{0}}\left(\frac{\bar{x}}{\varepsilon}\right)$, if $\bar{x} \in T_{\varepsilon}$, and $\psi_{\varepsilon}(\bar{x}):=0$, if $\bar{x} \in \omega \backslash T_{\varepsilon}$.

Using the definition of $\nu_{\varepsilon, 1}^{\tau}$, taking $\psi_{\varepsilon}$ as test function, using the uniform bounds in (1.1), the usual change of scales $\bar{y}=\varepsilon^{-1} \bar{x}$, together with the $Y$-periodicity of $\phi_{\gamma_{0}}$ and $\phi_{\varepsilon, 0}^{\tau}$, and since we have $\left\|\bar{\nabla} \phi_{\gamma_{0}}\right\|_{L^{\infty}(Y)} \leq c / \gamma_{0}$, we obtain

$$
0 \leq \nu_{\varepsilon, 1}^{\tau} \leq \frac{\eta}{\varepsilon^{2}} \frac{\int_{T_{\varepsilon}}\left[\phi_{\varepsilon, 0}^{\tau}\left(\frac{\bar{x}}{\varepsilon}\right)\right]^{2}\left|\left(\bar{\nabla} \phi_{\gamma_{0}}\right)\left(\frac{\bar{x}}{\varepsilon}\right)\right|^{2} \mathrm{~d} \bar{x}}{\int_{T_{\varepsilon}}\left[\phi_{\varepsilon, 0}^{\tau}\left(\frac{\bar{x}}{\varepsilon}\right)\right]^{2}\left|\phi_{\gamma_{0}}\left(\frac{\bar{x}}{\varepsilon}\right)\right|^{2} \mathrm{~d} \bar{x}} \leq \frac{\eta c^{2}}{\varepsilon^{2} \gamma_{0}^{2}} \frac{\int_{Q_{2 \gamma_{0}} \backslash Q_{\gamma_{0}}}\left|\phi_{\varepsilon, 0}^{\tau}(\bar{y})\right|^{2} \mathrm{~d} \bar{y}}{\int_{Q}\left|\phi_{\varepsilon, 0}^{\tau}(\bar{y})\right|^{2} \mathrm{~d} \bar{y}} .
$$

Using Lemma 5.2 with $k=n$ and recalling the definitions and the estimates therein, we obtain, for $\bar{y}=\bar{y}(s, \theta) \in$ $Q_{2 \gamma_{0}} \backslash Q$,

$$
\phi_{\varepsilon, 0}^{\tau}(\bar{y}(s, \theta))=\varepsilon^{\tau-1} P(s, \theta)+r_{k, \varepsilon}
$$

where, since $\tau \geq(k+2) / k$,

$$
\left\|r_{k, \varepsilon}\right\|_{L^{2}(Y \backslash Q)} \leq \bar{c}_{k} \varepsilon^{(\tau-1)\left(k+\frac{1}{2}\right)} \leq \bar{c}_{k} \varepsilon^{2+\frac{1}{k}}
$$

and $P$ satisfies the following pointwise estimate

$$
|P(s, \theta)|^{2} \leq \sum_{m=1}^{k} a_{m}\left(\frac{s}{\varepsilon}\right)^{j_{m}} \mathrm{e}^{-b_{m} \frac{s}{\varepsilon}}
$$

for some positive constants $a_{m}, b_{m}$ and for some $j_{m} \in \mathbb{N}$, independents of $\varepsilon$.

Consequently, putting together (5.28), (5.29) and (5.30), and in view of (5.27), we conclude that

$$
\begin{aligned}
0 \leq \nu_{\varepsilon, 1}^{\tau} \leq & \frac{\bar{c}}{\varepsilon^{2}}\left(\int_{Q}\left|\phi_{\varepsilon, 0}^{\tau}(\bar{y})\right|^{2} \mathrm{~d} \bar{y}\right)^{-1} \sum_{m=1}^{k} a_{m} \varepsilon^{\tau-1-j_{m}} \mathrm{e}^{-b_{m} \frac{\gamma_{0}}{\varepsilon}} \int_{Q_{2 \gamma_{0} \backslash Q_{\gamma_{0}}}} s^{j_{m}} \mathrm{~d} s \\
& +\frac{\bar{c}}{\varepsilon^{2}}\left(\int_{Q}\left|\phi_{\varepsilon, 0}^{\tau}(\bar{y})\right|^{2} \mathrm{~d} \bar{y}\right)^{-1} \varepsilon^{2\left(2+\frac{1}{k}\right)},
\end{aligned}
$$

for some constant $\bar{c}$ independent of $\varepsilon$. Having in mind that, from Lemma 5.1, $\int_{Q}\left|\phi_{\varepsilon, 0}^{\tau}(\bar{y})\right|^{2} \mathrm{~d} \bar{y} \rightarrow 1$ as $\varepsilon \rightarrow 0^{+}$, we may pass in (5.31) to the limit as $\varepsilon \rightarrow 0^{+}$to conclude the proof of Theorem 1.4.

Proof of Theorem 1.8. We start by observing that we may assume without loss of generality that $0 \in \omega$.

In view of the definition of $\lambda_{\varepsilon, 1}$ (see (5.26)), we deduce that

$$
\lambda_{\varepsilon, 1} \geq \frac{a_{\min }}{\varepsilon^{2 \tau}} \pi^{2},
$$

and so

$$
\lim _{\varepsilon \rightarrow 0^{+}}\left(\varepsilon^{2 \tau} \sigma_{\varepsilon}\right) \subset\left[a_{\min } \pi^{2},+\infty\right] .
$$

To prove the opposite inclusion we fix $\varepsilon>0$ and recall the notations of Proposition 2.5 with $B, b$ and $\lambda_{k}^{(n)}$ replaced by $\bar{A}_{\varepsilon}, \frac{a_{33}^{\varepsilon}}{\varepsilon^{2 \tau}}$ and $\lambda_{\varepsilon, k}^{(n)}$, respectively. Let also $\sigma_{\varepsilon}^{(n)}:=\left\{\lambda_{\varepsilon, k}^{(n)}: n \in \mathbb{N}\right\}$. 
For fixed $\varepsilon>0$ we have that $\varepsilon^{2 \tau} \lambda_{\varepsilon, k}^{(1)} \rightarrow+\infty$ as $k \rightarrow \infty$. Using a diagonal argument we can find a sequence $\left\{\lambda_{\varepsilon}\right\}_{\varepsilon>0} \subset \sigma_{\varepsilon}$ such that $\lambda_{\varepsilon} \rightarrow+\infty$ as $\varepsilon \rightarrow 0^{+}$. Thus,

$$
+\infty \in \lim _{\varepsilon \rightarrow 0^{+}}\left(\varepsilon^{2 \tau} \sigma_{\varepsilon}\right) .
$$

Moreover, by Proposition 2.5 one has

$$
\sigma_{\varepsilon}=\bigcup_{n \in \mathbb{N}} \sigma_{\varepsilon}^{(n)}
$$

We claim that

$$
\lim _{\varepsilon \rightarrow 0^{+}}\left(\varepsilon^{2 \tau} \sigma_{\varepsilon}^{(1)}\right) \supset\left[a_{\min } \pi^{2},+\infty\right) .
$$

Assume that (5.34) holds. Then the inclusion $\sigma_{\varepsilon} \supset \sigma_{\varepsilon}^{(1)}$ yields

$$
\lim _{\varepsilon \rightarrow 0^{+}}\left(\varepsilon^{2 \tau} \sigma_{\varepsilon}\right) \supset \lim _{\varepsilon \rightarrow 0^{+}}\left(\varepsilon^{2 \tau} \sigma_{\varepsilon}^{(1)}\right) \supset\left[a_{\min } \pi^{2},+\infty\right),
$$

which, together with (5.32) and (5.33), establishes (1.14).

In order to show (5.34) we first perform a change of variables that will transform problem

$$
\left\{\begin{array}{l}
-\operatorname{div}\left(\bar{A}_{\varepsilon}(\bar{x}) \bar{\nabla} \varphi_{\varepsilon}^{(1)}\right)+\frac{a_{33}^{\varepsilon}(\bar{x})}{\varepsilon^{2 \tau}} \pi^{2} \varphi_{\varepsilon}^{(1)}=\lambda_{\varepsilon}^{(1)} \varphi_{\varepsilon}^{(1)}, \text { a.e. } \bar{x} \in \omega, \\
\varphi_{\varepsilon} \in H_{0}^{1}(\omega)
\end{array}\right.
$$

into an equivalent one allowing us to pass to the limit as $\varepsilon \rightarrow 0^{+}$. Recall that problem (5.35) corresponds to $(2.10)$ for $n=1$, with $B$ replaced by $\bar{A}_{\varepsilon}$ and $b$ replaced by $\frac{a_{33}^{\varepsilon}}{\varepsilon^{2 \tau}}$.

Let $\omega_{\varepsilon}:=\frac{\omega}{\varepsilon^{\tau}}-\frac{\bar{y}_{0}}{\varepsilon^{\tau-1}}$, where $\bar{y}_{0}$ is a point of minimum of $a_{33}$. Notice that if $B \subset \mathbb{R}^{2}$ is a bounded set, then for all $\varepsilon>0$ small enough, $B \subset \omega_{\varepsilon}$, since $0 \in \omega$ and $\tau>1$. Associating to each function $\varphi \in H_{0}^{1}(\omega)$ the function $\psi \in H_{0}^{1}\left(\omega_{\varepsilon}\right)$ defined by $\psi(\bar{z}):=\varphi\left(\varepsilon^{\tau} \bar{z}+\varepsilon \bar{y}_{0}\right)$ and using the change of variables $\bar{z}:=\varepsilon^{-\tau} \bar{x}-\varepsilon^{1-\tau} \bar{y}_{0}$, (5.35) becomes

$$
\left\{\begin{array}{l}
-\operatorname{div}\left(\bar{D}_{\varepsilon}(\bar{z}) \bar{\nabla} \psi_{\varepsilon}^{(1)}\right)+d_{\varepsilon}(\bar{z}) \psi_{\varepsilon}^{(1)}=\rho_{\varepsilon}^{(1)} \psi_{\varepsilon}^{(1)}, \text { a.e. } \bar{z} \in \omega_{\varepsilon}, \\
\psi_{\varepsilon}^{(1)} \in H_{0}^{1}\left(\omega_{\varepsilon}\right),
\end{array}\right.
$$

where $\rho_{\varepsilon}^{(1)}:=\varepsilon^{2 \tau} \lambda_{\varepsilon}^{(1)}$, while $\bar{D}_{\varepsilon}$ and $d_{\varepsilon}$ are defined by

$$
\bar{D}_{\varepsilon}(\bar{z}):=\bar{A}\left(\varepsilon^{\tau-1} \bar{z}+\bar{y}_{0}\right), \quad d_{\varepsilon}(\bar{z}):=a_{33}\left(\varepsilon^{\tau-1} \bar{z}+\bar{y}_{0}\right) \pi^{2}, \quad \bar{z} \in \mathbb{R}^{2},
$$

respectively. In view of (1.1), for all $\xi \in \mathbb{R}^{2}$ and for a.e. $\bar{z} \in \mathbb{R}^{2}$ one has

$$
\zeta\|\xi\|^{2} \leq\left(\bar{D}_{\varepsilon}(\bar{z}) \xi \mid \xi\right) \leq \eta\|\xi\|^{2}, \quad \zeta \leq d_{\varepsilon}(\bar{z}) \leq \eta .
$$

Hence, up to a subsequence that we do not relabel, the sequence $\left\{\bar{D}_{\varepsilon}\right\}_{\varepsilon>0} G$-converges to some matrix $\bar{D}_{0}$ (see $[7,15])$ and the sequence $\left\{d_{\varepsilon}\right\}_{\varepsilon>0}$ weakly- $\star$ converges in $L^{\infty}\left(\mathbb{R}^{2}\right)$ to some $d_{0} \in L^{\infty}\left(\mathbb{R}^{2}\right)$. On the other hand, since $a_{\alpha \beta}$ and $a_{33}$ are continuous in a neighborhood of $\bar{y}_{0}, \bar{D}_{\varepsilon} \rightarrow \bar{A}\left(\bar{y}_{0}\right)$ and $d_{\varepsilon} \rightarrow a_{33}\left(\bar{y}_{0}\right) \pi^{2}$ uniformly on each compact subset of $\mathbb{R}^{2}$ as $\varepsilon \rightarrow 0^{+}$. Thus, by definition of $H$-limit, we conclude that $\bar{D}_{0} \equiv \bar{A}\left(\bar{y}_{0}\right)$ and $d_{0} \equiv a_{33}\left(\bar{y}_{0}\right) \pi^{2}=a_{\min } \pi^{2}$. In particular, the whole sequences $\left\{\bar{D}_{\varepsilon}\right\}_{\varepsilon>0}$ and $\left\{d_{\varepsilon}\right\}_{\varepsilon>0}$ converge.

Let $\mathcal{S}_{\varepsilon}$ represent the self-adjoint operator $-\operatorname{div}\left(\bar{D}_{\varepsilon} \bar{\nabla} \cdot\right)+d_{\varepsilon} \cdot$ from $L^{2}\left(\omega_{\varepsilon}\right)$ into itself. Then its spectrum is $\sigma\left(\mathcal{S}_{\varepsilon}\right)=\varepsilon^{2 \tau} \sigma_{\varepsilon}^{(1)}$. Therefore, proving (5.34) is equivalent to proving

$$
\lim _{\varepsilon \rightarrow 0^{+}} \sigma\left(\mathcal{S}_{\varepsilon}\right) \supset\left[d_{0},+\infty\right) .
$$


Consider now the inverse operator, $\mathcal{S}_{\varepsilon}^{-1}$, of $\mathcal{S}_{\varepsilon}$, i.e. the compact self-adjoint operator from $L^{2}\left(\omega_{\varepsilon}\right)$ into itself that associates to each $f_{\varepsilon} \in L^{2}\left(\omega_{\varepsilon}\right), \mathcal{S}_{\varepsilon}^{-1} f_{\varepsilon}:=\psi_{\varepsilon}$, where $\psi_{\varepsilon} \in H_{0}^{1}\left(\omega_{\varepsilon}\right)$ is the solution of

$$
\left\{\begin{array}{l}
-\operatorname{div}\left(\bar{D}_{\varepsilon} \bar{\nabla} \psi_{\varepsilon}\right)+d_{\varepsilon} \psi_{\varepsilon}=f_{\varepsilon}, \text { a.e. in } \omega_{\varepsilon} \\
\psi_{\varepsilon} \in H_{0}^{1}\left(\omega_{\varepsilon}\right)
\end{array}\right.
$$

For the sake of simplicity we will not distinguish a function in $H_{0}^{1}\left(\omega_{\varepsilon}\right)$ from its zero extension to the whole $\mathbb{R}^{2}$.

Let us also introduce the self-adjoint operators from $L^{2}\left(\mathbb{R}^{2}\right)$ into itself, $\mathcal{S}:=-\operatorname{div}\left(\bar{D}_{0} \bar{\nabla} \cdot\right)+d_{0}$. and its inverse operator $\mathcal{S}^{-1}$, that associates to each $f \in L^{2}\left(\mathbb{R}^{2}\right), \mathcal{S}^{-1} f:=\psi$, where $\psi \in H^{1}\left(\mathbb{R}^{2}\right)$ is the solution of

$$
\left\{\begin{array}{l}
-\operatorname{div}\left(\bar{D}_{0} \bar{\nabla} \psi\right)+d_{0} \psi=f, \text { a.e. in } \mathbb{R}^{2}, \\
\psi \in H^{1}\left(\mathbb{R}^{2}\right)
\end{array}\right.
$$

Since $\bar{D}_{0}$ is a positive definite constant matrix and $d_{0}>0, \sigma(S)=\left[d_{0},+\infty\right)$. Hence, if we prove that

$$
\lim _{\varepsilon \rightarrow 0^{+}} \sigma\left(\mathcal{S}_{\varepsilon}^{-1}\right) \supset \sigma\left(\mathcal{S}^{-1}\right),
$$

it follows that $\lim _{\varepsilon \rightarrow 0^{+}} \sigma\left(\mathcal{S}_{\varepsilon}\right) \supset \sigma(\mathcal{S})=\left[d_{0},+\infty\right)$, which is precisely (5.39). In order to show (5.42), we start by proving that $\mathcal{S}_{\varepsilon}^{-1}$ converges strongly to $\mathcal{S}^{-1}$ as $\varepsilon \rightarrow 0^{+}$; more precisely, if $f \in L^{2}\left(\mathbb{R}^{2}\right)$, then $\mathcal{S}_{\varepsilon}^{-1} f \chi_{\omega_{\varepsilon}} \rightarrow \mathcal{S}^{-1} f$ in $L^{2}\left(\mathbb{R}^{2}\right)$ as $\varepsilon \rightarrow 0^{+}$.

Let $f \in L^{2}\left(\mathbb{R}^{2}\right)$, and define $f_{\varepsilon}:=f \chi_{\omega_{\varepsilon}} \in L^{2}\left(\omega_{\varepsilon}\right)$. Let $\psi_{\varepsilon}:=\mathcal{S}_{\varepsilon}^{-1} f_{\varepsilon}$ (extended by zero outside $\omega_{\varepsilon}$ ) and $\psi:=\mathcal{S}^{-1} f$. Thanks to (5.38), we have, up to a subsequence that we do not relabel, $\psi_{\varepsilon} \rightarrow \varphi$ weakly in $H^{1}\left(\mathbb{R}^{2}\right)$ as $\varepsilon \rightarrow 0^{+}$, for some $\varphi \in H^{1}\left(\mathbb{R}^{2}\right)$. Moreover, since $\psi_{\varepsilon}$ is the solution of (5.40), if $\vartheta \in C_{c}^{\infty}\left(\mathbb{R}^{2}\right)$ then we have, for all $\varepsilon>0$ small enough, $\operatorname{supp} \vartheta \subset \omega_{\varepsilon}$ and

$$
\int_{\mathbb{R}^{2}} \overline{D_{\varepsilon}} \bar{\nabla} \psi_{\varepsilon} \bar{\nabla} \vartheta \mathrm{d} \bar{z}+\int_{\mathbb{R}^{2}} d_{\varepsilon} \psi_{\varepsilon} \vartheta \mathrm{d} \bar{z}=\int_{\mathbb{R}^{2}} f \vartheta \mathrm{d} \bar{z}
$$

Letting $\varepsilon \rightarrow 0^{+}$we obtain

$$
\int_{\mathbb{R}^{2}} \bar{D}_{0} \bar{\nabla} \varphi \bar{\nabla} \vartheta \mathrm{d} \bar{z}+\int_{\mathbb{R}^{2}} d_{0} \varphi \vartheta \mathrm{d} \bar{z}=\int_{\mathbb{R}^{2}} f \vartheta \mathrm{d} \bar{z} .
$$

Since $\vartheta \in C_{c}^{\infty}\left(\mathbb{R}^{2}\right)$ was arbitrary, we deduce that $\varphi=\psi$ a.e. in $\mathbb{R}^{2}$. Thus, $\psi_{\varepsilon} \rightarrow \psi$ weakly in $H^{1}\left(\mathbb{R}^{2}\right)$ as $\varepsilon \rightarrow 0^{+}$ and so, to establish the strong convergence in $L^{2}\left(\mathbb{R}^{2}\right)$ it suffices to prove that

$$
\lim _{\varepsilon \rightarrow 0^{+}} \int_{\mathbb{R}^{2}}\left|\psi_{\varepsilon}\right|^{2} \mathrm{~d} \bar{z}=\int_{\mathbb{R}^{2}}|\psi|^{2} \mathrm{~d} \bar{z} .
$$

Let $L:=\liminf _{\varepsilon \rightarrow 0^{+}} \int_{\mathbb{R}^{2}}\left|\psi_{\varepsilon}\right|^{2} \mathrm{~d} \bar{z}$. Without loss of generality we may assume that the inferior limit defining $L$ is actually a limit, otherwise we would extract a subsequence. By the sequential lower semicontinuity of the norm with respect to the weak topology of $L^{2}\left(\mathbb{R}^{2}\right), L \geq \int_{\mathbb{R}^{2}}|\psi|^{2} \mathrm{~d} \bar{z}$.

To prove the converse inequality, we start by observing that in view of [7], Theorem 13.12, if $c \in \mathbb{R}$ is such that $\inf _{\varepsilon} d_{\varepsilon}>c>0$, then the sequence of functionals $\left\{F_{\varepsilon}\right\}_{\varepsilon>0}$, where $F_{\varepsilon}: H^{1}\left(\mathbb{R}^{2}\right) \rightarrow[0,+\infty]$ is defined by

$$
F_{\varepsilon}(v):= \begin{cases}\int_{\omega_{\varepsilon}} \bar{D}_{\varepsilon}(\bar{z}) \bar{\nabla} v(\bar{z}) \bar{\nabla} v(\bar{z})+\left(d_{\varepsilon}(\bar{z})-c\right)|v(\bar{z})|^{2} \mathrm{~d} \bar{z}, & \text { if } v \in H_{0}^{1}\left(\omega_{\varepsilon}\right), \\ +\infty, & \text { otherwise, }\end{cases}
$$

$\Gamma$-converges as $\varepsilon \rightarrow 0^{+}$, with respect to the weak topology of $H^{1}\left(\mathbb{R}^{2}\right)$, to the functional $F_{0}: H^{1}\left(\mathbb{R}^{2}\right) \rightarrow[0,+\infty]$ given by

$$
F_{0}(v):=\int_{\mathbb{R}^{2}} \bar{D}_{0} \bar{\nabla} v(\bar{z}) \bar{\nabla} v(\bar{z})+\left(d_{0}-c\right)|v(\bar{z})|^{2} \mathrm{~d} \bar{z} .
$$


Thus, since $\psi_{\varepsilon} \rightarrow \psi$ in $H^{1}\left(\mathbb{R}^{2}\right)$ as $\varepsilon \rightarrow 0^{+}$,

$$
\liminf _{\varepsilon \rightarrow 0^{+}} \int_{\omega_{\varepsilon}} \bar{D}_{\varepsilon} \bar{\nabla} \psi_{\varepsilon} \bar{\nabla} \psi_{\varepsilon}+\left(d_{\varepsilon}-\bar{\zeta}\right)\left|\psi_{\varepsilon}\right|^{2} \mathrm{~d} \bar{z} \geq \int_{\mathbb{R}^{2}} \bar{D}_{0} \bar{\nabla} \psi \bar{\nabla} \psi+\left(d_{0}-\bar{\zeta}\right)|\psi|^{2} \mathrm{~d} \bar{z},
$$

for any $0<\bar{\zeta}<\zeta$. Furthermore, using, in addition, the strong convergence $f_{\varepsilon} \rightarrow f$ in $L^{2}\left(\mathbb{R}^{2}\right)$ as $\varepsilon \rightarrow 0^{+}$, and the fact that (5.43) holds for all $\vartheta \in H_{0}^{1}\left(\omega_{\varepsilon}\right)$ and (5.44) holds for all $\vartheta \in H^{1}\left(\mathbb{R}^{2}\right)$, we deduce that

$$
\int_{\omega_{\varepsilon}} \bar{D}_{\varepsilon} \bar{\nabla} \psi_{\varepsilon} \bar{\nabla} \psi_{\varepsilon}+d_{\varepsilon}\left|\psi_{\varepsilon}\right|^{2} \mathrm{~d} \bar{z}=\int_{\omega_{\varepsilon}} f \psi_{\varepsilon} \mathrm{d} \bar{z} \underset{\varepsilon \rightarrow 0^{+}}{\longrightarrow} \int_{\mathbb{R}^{2}} f \psi \mathrm{d} \bar{z}=\int_{\mathbb{R}^{2}} \bar{D}_{0} \bar{\nabla} \psi \bar{\nabla} \psi+d_{0}|\psi|^{2} \mathrm{~d} \bar{z} .
$$

Consequently,

$$
\begin{aligned}
\liminf _{\varepsilon \rightarrow 0^{+}} \int_{\omega_{\varepsilon}} \bar{D}_{\varepsilon} \bar{\nabla} \psi_{\varepsilon} \bar{\nabla} \psi_{\varepsilon}+\left(d_{\varepsilon}-\bar{\zeta}\right)\left|\psi_{\varepsilon}\right|^{2} \mathrm{~d} \bar{z} & =\lim _{\varepsilon \rightarrow 0^{+}}\left(\int_{\omega_{\varepsilon}} \bar{D}_{\varepsilon} \bar{\nabla} \psi_{\varepsilon} \bar{\nabla} \psi_{\varepsilon}+d_{\varepsilon}\left|\psi_{\varepsilon}\right|^{2} \mathrm{~d} \bar{z}\right)-\bar{\zeta} L \\
& =\int_{\mathbb{R}^{2}} \bar{D}_{0} \bar{\nabla} \psi \bar{\nabla} \psi+d_{0}|\psi|^{2} \mathrm{~d} \bar{z}-\bar{\zeta} L,
\end{aligned}
$$

where we also used the definition of $L$. From (5.45) and (5.46) we deduce that $L \leq \int_{\mathbb{R}^{2}}|\psi|^{2} \mathrm{~d} \bar{z}$. Hence, $L=\int_{\mathbb{R}^{2}}|\psi|^{2} \mathrm{~d} \bar{z}$ and $\mathcal{S}_{\varepsilon}^{-1} f_{\varepsilon}=\psi_{\varepsilon} \rightarrow \psi=\mathcal{S}^{-1} f$ in $L^{2}\left(\mathbb{R}^{2}\right)$ as $\varepsilon \rightarrow 0^{+}$.

Finally, we prove (5.42). Assume by contradiction that there is $\gamma \in \sigma\left(\mathcal{S}^{-1}\right)$ which is not a cluster point of $\sigma\left(\mathcal{S}_{\varepsilon}^{-1}\right)$. Then there exist $c>0$ and $\varepsilon_{0}>0$ such that for all $\gamma_{\varepsilon} \in \sigma\left(\mathcal{S}_{\varepsilon}^{-1}\right)$ with $0<\varepsilon<\varepsilon_{0}$ one has

$$
\left|\gamma_{\varepsilon}-\gamma\right|>c .
$$

Let $f \in L^{2}\left(\mathbb{R}^{2}\right)$, and set $f_{\varepsilon}:=f \chi_{\omega_{\varepsilon}} \in L^{2}\left(\omega_{\varepsilon}\right)$. If $\gamma_{\varepsilon} \in \sigma\left(\mathcal{S}_{\varepsilon}^{-1}\right)$ with $0<\varepsilon<\varepsilon_{0}$, then

$$
\left\|\mathcal{S}_{\varepsilon}^{-1} f_{\varepsilon}-\gamma f_{\varepsilon}\right\|_{L^{2}\left(\mathbb{R}^{2}\right)}=\left\|\mathcal{S}_{\varepsilon}^{-1} f_{\varepsilon}-\gamma f_{\varepsilon}\right\|_{L^{2}\left(\omega_{\varepsilon}\right)} \geq\left|\gamma_{\varepsilon}-\gamma\right|\left\|f_{\varepsilon}\right\|_{L^{2}\left(\omega_{\varepsilon}\right)} \geq c\left\|f_{\varepsilon}\right\|_{L^{2}\left(\mathbb{R}^{2}\right)} .
$$

Using the strong convergence of $\mathcal{S}_{\varepsilon}^{-1}$ established above together with the strong convergence $f_{\varepsilon} \rightarrow f$ in $L^{2}\left(\mathbb{R}^{2}\right)$ as $\varepsilon \rightarrow 0^{+}$, and letting $\varepsilon \rightarrow 0^{+}$in (5.47), we get

$$
\left\|\mathcal{S}^{-1} f-\gamma f\right\|_{L^{2}\left(\mathbb{R}^{2}\right)} \geq c\|f\|_{L^{2}\left(\mathbb{R}^{2}\right)},
$$

which contradicts the fact that $\gamma \in \sigma\left(\mathcal{S}^{-1}\right)$ since $f \in L^{2}\left(\mathbb{R}^{2}\right)$ was taken arbitrarily. Thus (5.42) holds, and this finishes the proof of Theorem 1.8.

\section{A. Appendix}

We prove the results announced in Remark 1.3. From (1.9) we obtain that $\left|\rho_{j}\right| \leq C\left\|\psi_{j}\right\|_{L^{2}(Y)}$, where $C$ is a constant independent of $j$. On the other hand, the sum on the right hand side of (1.10) is the general term of the Cauchy convolution of the series $\psi:=\sum_{j \geqslant 0} \psi_{j}$ and $\rho:=\sum_{j \geqslant 0} \rho_{j}$. Summing (1.10) in $j \geq 0$ and passing to the limit, we get

$$
\left\{\begin{array}{l}
-\operatorname{div}(\bar{A} \bar{\nabla} \psi)+a_{33} \pi^{2} \psi=\rho \psi, \text { a.e. in } Y, \\
\psi \in H_{\#}^{1}(Y), \int_{Y} \psi \mathrm{d} \bar{y}=1,
\end{array}\right.
$$

which implies $\psi=\phi_{0} / \int_{Y} \phi_{0} \mathrm{~d} \bar{y}$ and $\rho=\mu_{0}$ (see (1.6) for $\tau=1$ ). Finally, in order to obtain the convergence as $\tau \rightarrow 1^{-}$, we fix $\varepsilon>0$ and consider, for an arbitrary $\delta>0, j_{0} \in \mathbb{N}$ such that $\sum_{j>j_{0}}\left|\rho_{j}\right|<\delta$. As $\tau$ 
is of order $\frac{i}{i+1}$, to obtain the desired convergence it is enough to prove that

$$
\lim _{i \rightarrow+\infty} \sum_{j=0}^{i} \varepsilon^{\frac{2(j+1)}{i+1}} \rho_{j}=\rho=\mu_{0}
$$

Since $\sum_{j=0}^{i} \varepsilon^{\frac{2(j+1)}{i+1}} \rho_{j}=\sum_{j=0}^{j_{0}} \varepsilon^{\frac{2(j+1)}{i+1}} \rho_{j}+\sum_{j=j_{0}+1}^{i} \varepsilon^{\frac{2(j+1)}{i+1}} \rho_{j},\left|\sum_{j=j_{0}+1}^{i} \varepsilon^{\frac{2(j+1)}{i+1}} \rho_{j}\right| \leq \sum_{j>j_{0}}\left|\rho_{j}\right| \leq \delta$ and $\lim _{i \rightarrow+\infty} \sum_{j=0}^{j_{0}} \varepsilon^{\frac{2(j+1)}{i+1}} \rho_{j}=$ $\sum_{j=0}^{j_{0}} \rho_{j}$, the arbitrariness of $\delta$ yields (A.1).

Acknowledgements. The work of R. Ferreira was supported by the Fundação para a Ciência e a Tecnologia (Portuguese Foundation for Science and Technology) through the Carnegie Mellon Portugal Program under Grant SFRH/BD/35695/ 2007. The work of M.L. Mascarenhas was partially supported by the Fundação para a Ciência e a Tecnologia (Portuguese Foundation for Science and Technology) through the Financiamento Base 2009 ISFL-1-297 and through the Carnegie Mellon Portugal Program in Applied Mathematics.

\section{REFERENCES}

[1] G. Allaire and C. Conca, Bloch wave homogenization and spectral asymptotic analysis. J. Math. Pures Appl. 77 (1998) 153-208.

[2] G. Allaire and F. Malige, Analyse asymptotique spectrale d'un problème de diffusion neutronique. C. R. Acad. Sci. Paris, Ser. I 324 (1997) 939-944.

[3] H. Attouch, Variational convergence for functions and operators. Applicable Mathematics Series, Pitman (Advanced Publishing Program), Boston, MA (1984).

[4] N.S. Bachvalov and G.P. Panasenko, Homogenization of Processes in Periodic Media. Nauka, Moscow (1984).

[5] A. Bensoussan, J.-L. Lions and G. Papanicolaou, Asymptotic analysis for periodic structures. North-Holland Publishing Co., Amsterdam (1978)

[6] G. Bouchitté, M.L. Mascarenhas and L. Trabucho, On the curvature and torsion effects in one dimensional waveguides. ESAIM: COCV 13 (2007) 793-808.

[7] G. Dal Maso, An introduction to $\Gamma$-convergence, Progress in Nonlinear Differential Equations and their Applications 8. Birkhäuser Boston Inc., Boston (1993).

[8] R. Ferreira and M.L. Mascarenhas, Waves in a thin and periodically oscillating medium. C. R. Math. Acad. Sci. Paris, Ser. I 346 (2008) 579-584.

[9] D. Gilbarg and N. Trudinger, Elliptic partial differential equations of second order. Classics in Mathematics, Springer-Verlag, New York (1977).

[10] V. Jikov, S. Kozlov and O. Oleĭnik, Homogenization of differential operators and integral functionals. Springer-Verlag, Berlin (1994).

[11] S. Kesavan, Homogenization of Elliptic Eigenvalue Problems: Part 1. Appl. Math. Optim. 5 (1979) 153-167.

[12] S. Kesavan, Homogenization of Elliptic Eigenvalue Problems: Part 2. Appl. Math. Optim. 5 (1979) 197-216.

[13] S. Kozlov and A. Piatnitski, Effective diffusion for a parabolic operator with periodic potential. SIAM J. Appl. Math. 53 (1993) 401-418.

[14] S. Kozlov and A. Piatnitski, Degeneration of effective diffusion in the presence of periodic potential. Ann. Inst. H. Poincaré Probab. Statist. 32 (1996) 571-587.

[15] F. Murat and L. Tartar, H-Convergence, in Topics in the mathematical modelling of composite materials. Progr. Nonlinear Differential Equations Appl., Birkhäuser Boston, Boston (1997).

[16] O.A. Oleı̆nik, A.S. Shamaev and G.A. Yosifian, Mathematical problems in elasticity and homogenization. Studies in Mathematics and its Applications, North-Holland Publishing Co., Amsterdam (1992).

[17] M. Vanninathan, Homogenization of eigenvalue problems in perforated domains. Proc. Indian Acad. Sci. Math Sci. 90 (1981) 239-271.

[18] M.I. Vishik and L.A. Lyusternik, Regular degeneration and boundary layer for linear differential equations with small parameter. Amer. Math. Soc. Transl. (2) 20 (1962) 239-364 [English translation]. 